\title{
Stability and Open-Loop Dynamics of Very Flexible Aircraft Including Free-Wake Effects
}

\author{
J. Murua, H. Hesse, R. Palacios ${ }^{\dagger}$ J. M. R. Graham ${ }^{\ddagger}$ \\ Department of Aeronautics, Imperial College, London, SW7 2AZ, United Kingdom
}

\begin{abstract}
The paper investigates the coupled nonlinear aeroelasticity and flight mechanics of very flexible lightweight aircraft. A geometrically-exact composite beam formulation is used to model the nonlinear flexible-body dynamics, including rigid-body motions. The aerodynamics are modeled by a general 3-D unsteady vortex-lattice method, which can capture the instantaneous shape of the lifting surfaces and the free wake, including large displacements and interference effects. The coupled governing equations are solved in a variety of ways, allowing linear and nonlinear time-domain simulations of the full vehicle and frequencydomain linear stability analysis around trimmed configurations. The resulting framework for the Simulation of High-Aspect Ratio Planes (SHARP) provides a medium-fidelity representation of flexible aircraft dynamics, based on an intuitive and easily linearizable structural representation using displacements and the Cartesian rotation vector, time-domain aerodynamics, and at relatively low computational costs. Previous verification studies on the structural dynamics and aerodynamics modules are complemented here with studies on the flexible-body implementation and on the integrated simulation methodology. A numerical investigation is finally presented on a representative high-altitude long-endurance model aircraft, investigating its stability properties and its open-loop dynamic response.
\end{abstract}

\section{Nomenclature}

$\Delta b \quad$ spanwise dimension of aerodynamic vortex ring

$C \quad$ global tangent damping matrix

$C^{B a}$ coordinate transformation matrix, from $a$ to $B$

$\Delta c$ chordwise dimension of aerodynamic vortex ring

$\vec{F} \quad$ internal forces in a deformed beam section

$K \quad$ global tangent stiffness matrix

$m$ mass per unit length

$M \quad$ global tangent mass matrix

$\mathcal{M}$ cross-sectional mass matrix

$\vec{M} \quad$ internal moments in a deformed beam section

$\vec{p} \quad$ position vector of the origin of the body-fixed frame, $a$, from the inertial one, $G$

$Q \quad$ global vector generalized forces in the structural problem

$\vec{R} \quad$ local position vector along the beam reference line

$s \quad$ arc length along reference line of the beam elements

$\mathcal{S}$ cross-sectional stiffness matrix

$t$ physical time

$\vec{v} \quad$ inertial translational velocity of the body-fixed frame, $a$

$\vec{V} \quad$ inertial translation velocity at a beam location

$\boldsymbol{w} \quad$ vector of non-vortical induced velocity at all collocation points

$\boldsymbol{X}_{b} \quad$ coordinates of the aerodynamic lattice, expressed in the aerodynamic frame of reference

* Graduate student, AIAA Student Member.

$\dagger$ Lecturer, AIAA Member (Contact author. e-mail: rpalacio@imperial.ac.uk).

${ }^{\ddagger}$ Professor. 


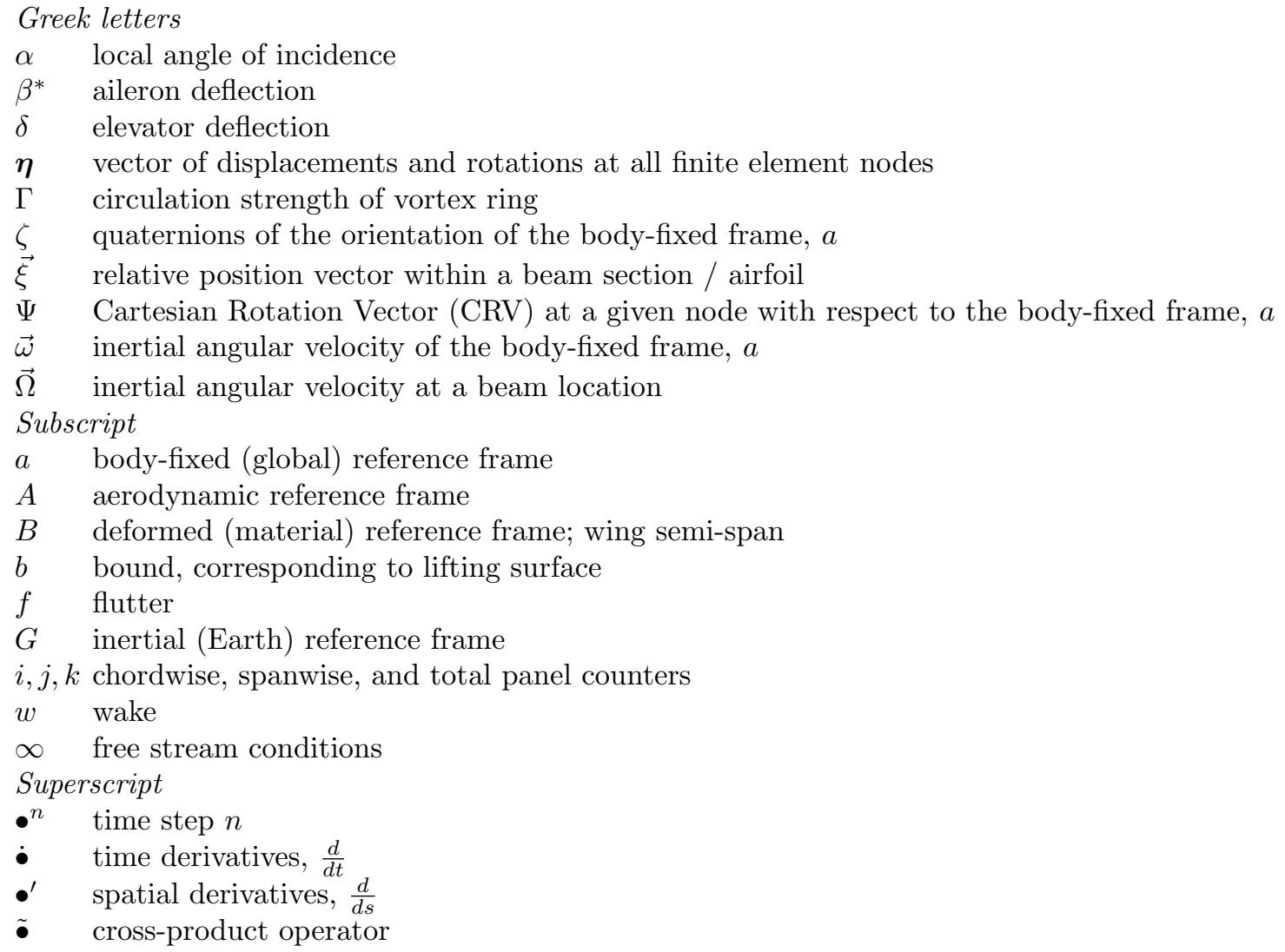

\section{Introduction}

Solar- and hydrogen-powered aircraft for very-long-endurance unmanned flight are finally a reality, and currently there are several prototypes in different stages of development at both sides of the Atlantic. Due to the exceptionally demanding efficiency requirements, these vehicles are built on an extremely light structure with large-aspect-ratio wings. This brings a number of issues that need to be considered in the design process: the possibility of large (geometrically-nonlinear) structural deformations, coupling between the aeroelastic and flight dynamics responses, low resilience to atmospheric turbulence and gusts, controllability issues, etc. New multidisciplinary design frameworks are needed for the analysis of these Very Flexible Aircraft (VFA) and the design of appropriate controllers, and a substantial research effort has been carried out towards this goal in recent years. ${ }^{1-6}$ In most cases, the characteristic slenderness of all primary flexible structures of VFA has motivated descriptions of the nonlinear structural dynamics through beam models and of the unsteady aerodynamics by means of 2-D strip theory. Three-dimensional aerodynamic effects may be important however in the interference between lifting surfaces (typically between the wing and tail) or in the wing tip effects. The latter is typically considered through corrections on the lift curve, but those are only valid in small ranges of reduced frequencies. ${ }^{7}$

Potential-flow-based 3-D aerodynamic models provide the next order of fidelity, without compromising the simplicity of the unified model or bringing a substantial computational burden. VFA are likely to satisfy potential-flow assumptions (low-speed flight and attached flow) during climb and descend operations, hence rendering these models excellent candidates for the mission segments in which significant gust encounters may occur. Among potential-flow solvers, free-wake methods become necessary for unsteady flow situations with complex wing kinematics (including large deformations), and for the investigation of interference phenomena, which is likely to impact VFA dynamics. The Unsteady Vortex Lattice Method (UVLM) has been shown ${ }^{8}$ to be a good candidate for, at least, global studies of strongly interfering flow fields, and this has been illustrated in a previous work by the authors ${ }^{9}$ on the impact of the wing wake on the tail aerodynamics. Conventional UVLM implementations are based on a $1^{\text {st }}$ order forward Euler explicit numerical scheme. ${ }^{10}$ While this simple approach usually gives satisfactory results, it poses restrictions on the stability and accuracy of the 
coupled model of the aircraft dynamics. In this work, we will formulate the UVLM in a more general way so that different time integration algorithms could be implemented. This would enable a tight coupling with the structural- and flight-dynamics, where all equations could be solved simultaneously, achieving higher fidelity and convergence properties. In addition, a tightly-coupled state-space description of the unified model would lead to a framework in which stability analysis, linear modal reduction and integration of control laws could be carried out in a straightforward manner.

In a previous work, ${ }^{7}$ we investigated and compared different independent solution procedures for the unsteady aerodynamics and structural dynamics response of VFA. Based on that work, this paper will present a unified model for the accurate prediction of the nonlinear dynamics of VFA. To that goal, a geometricallyexact composite beam finite-element model will be used to characterize the structural dynamics. The primary structural variables are the local displacements and the Cartesian rotation vector. Even though this last selection for the parameterization of the finite rotations yields a more complex algebra than other solution approaches, ${ }^{4,5}$ it was found to be a more intuitive approach and one from which linear solutions correspond to standard structural dynamics model, allowing easy comparison of the results. The rigid-body dynamics of the unsupported structure are captured by the translational and angular velocities of a body-fixed reference frame. The aerodynamic model is defined by the UVLM, which has been reformulated to accommodate more general integration algorithms. The full set of governing equations is finally formulated in a loosely-coupled nonlinear form, and in a closely-coupled discrete state-space fashion for linear analysis around nonlinearly deformed configurations.

\section{Aerodynamic Model: Unsteady Vortex Lattice Method}

The Unsteady Vortex Lattice Method (UVLM) is an effective computational technique to solve 3-D potential flow problems about lifting surfaces. The basics of the UVLM algorithm are described by Katz and Plotkin ${ }^{10}$ using an explicit time-stepping technique. As in other panel methods, such as the DoubletLattice, ${ }^{11,12}$ elementary (singularity) solutions are distributed over a surface and the non-penetration boundary condition is imposed at a number of control (collocation) points, leading to a system of algebraic equations. The UVLM is based on thin-airfoil approximation, so both the elementary solutions and the collocation points are placed over the instantaneous (i.e., deformed) mean surface in lieu of the actual surface, thus effectively ignoring thickness effects.

Lifting surfaces and wakes (the latter obtained as part of the solution procedure) are discretized using vortex rings, i.e., quadrilateral elements composed by discrete vortex segments in a closed loop, along which the circulation strength, $\Gamma_{k}$, is constant (see Figure 1). At the beginning of the motion, only the vortex rings covering the lifting surface exist (bound elements), and collocation points at which flow-tangency will be imposed are associated to them. As the surface moves along its flight path, a force-free wake is formed, shed and convected. In order to accomplish this, each trailing edge bound vortex ring sheds a wake panel, with circulation equal to the trailing edge ring strength at the previous time step. The wake elements are then freely moved according to the local flow velocity, and thus generate a vortex ring lattice representing the shed wake.

In the absence of dissipation, the circulation strength of the wake rings remains constant as they move away from the lifting surface, but they are allowed to roll up and stretch. Albeit dissipation could also be accounted for by implementing any of various models for wake decay, ${ }^{13,14}$ this was not regarded as necessary for the applications under study, since the influence of the wake already decays very rapidly as it is convected away from the lifting surface. Benefiting from this, the computational burden can be significantly reduced by neglecting the influence of very far wake panels and truncating the wake.

The circulation strength of the bound vortex elements is determined by applying the non-penetration boundary condition, requiring a zero velocity component normal to the solid surface at every collocation point $k$. From this distribution of vortex elements, the aerodynamic loads acting on the lifting surface can be computed using the unsteady Bernoulli equation.

In this section, the discrete-time equations governing the UVLM are presented. The aerodynamic states that fully define the problem, in the most general case, are

$$
\boldsymbol{x}_{A}=\left[\begin{array}{llll}
\boldsymbol{\Gamma}_{b}^{T} & \boldsymbol{\Gamma}_{w}^{T} & \dot{\boldsymbol{\Gamma}}_{b}^{T} & \boldsymbol{X}_{w}^{T}
\end{array}\right]^{T},
$$

where $\boldsymbol{\Gamma}_{b}$ and $\boldsymbol{\Gamma}_{w}$ are the vectors with the circulation strengths in the bound and wake vortex rings, respectively, $\dot{\boldsymbol{\Gamma}}_{b}$ represents the derivative of bound circulations and $\boldsymbol{X}_{w}$ is the vector with the wake grid-coordinates, 


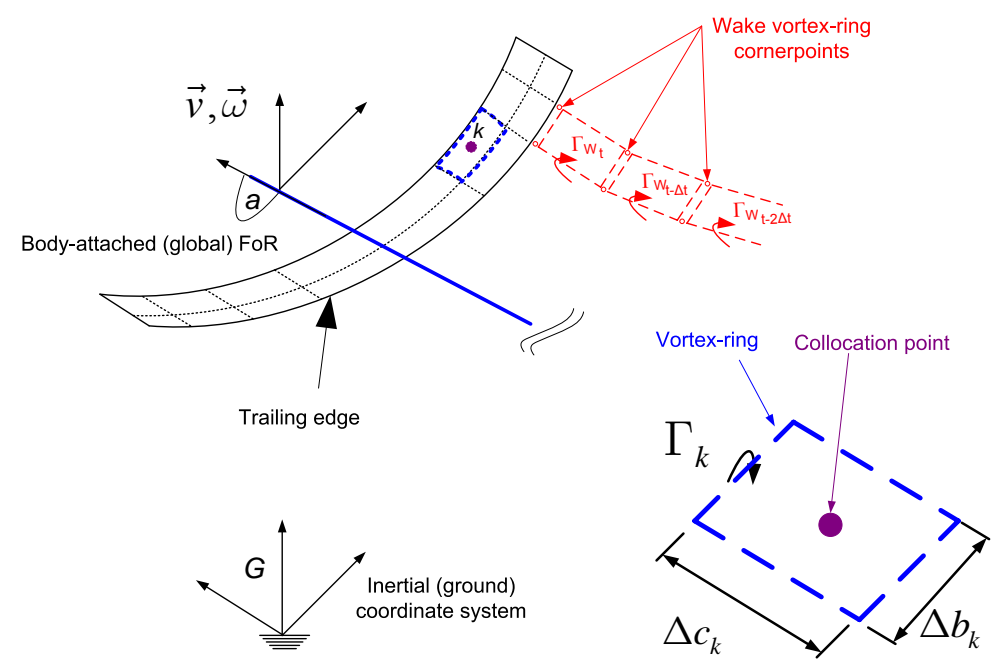

Figure 1. Unsteady aerodynamics model: lifting surface and wake discretization using vortex ring elements.

thus defining the wake shape. At discrete time step $n+1$, the non-penetration boundary condition can be formulated as

$$
A_{b} \boldsymbol{\Gamma}_{b}^{n+1}+A_{w} \boldsymbol{\Gamma}_{w}^{n+1}+\boldsymbol{w}^{n+1}=0,
$$

where $A_{b}=A_{b}\left(\boldsymbol{X}_{b}^{n+\epsilon}\right)$ and $A_{w}=A_{w}\left(\boldsymbol{X}_{b}^{n+\epsilon}, \boldsymbol{X}_{w}^{n+\epsilon}\right)$ are the wing-wing and wing-wake aerodynamic influence coefficient matrices, respectively, and $\boldsymbol{X}_{b}$ includes the coordinates of the bound vortex-rings. Elements of these matrices are obtained by projecting the velocity computed using the Biot-Savart law over the vortexring normal vector, and the time at which they are evaluated within the current time step, determined by $0 \leq \epsilon \leq 1$, depends on the integration scheme. The last term in Eq. (2) is the vector of normal components of the non-vortical induced velocities at the collocation points, and may include gust-induced velocities, wing deformations and rigid-body motions. We will write it as

$$
\boldsymbol{w}^{n+1}=W_{b}\left(\dot{\boldsymbol{X}}_{b}^{n+1}+\boldsymbol{u}_{g}^{n+1}\right)
$$

where $W_{b}\left(\boldsymbol{X}_{b}^{n+\epsilon}\right)$ is a matrix that projects the local velocities along the normal direction to the panels, and $\boldsymbol{u}_{g}$ is the vector with local gust velocities (if present). For a purely aerodynamic problem, that is, in which neither structural dynamics nor flight dynamics are solved, the motion of the lifting surfaces will be prescribed and in this case, $\boldsymbol{X}_{b}$ and $\dot{\boldsymbol{X}}_{b}$ will be part of the inputs to the system. If a coupled aeroelastic and flight dynamics problem is considered, $\boldsymbol{X}_{b}$ and $\dot{\boldsymbol{X}}_{b}$ will be a function of the structural and rigid-body states, as described in section IV, as well as including inputs such as deployment of control surfaces.

At each time step, a new row of vortex rings will be shed into the wake from the trailing edge of each lifting surface. In addition to this, the existing wake will displace following the local flow velocity (the free wake model). This is written as

$$
\boldsymbol{X}_{w}^{n+1}=C_{b} \boldsymbol{X}_{b}^{n+1}+C_{w} \boldsymbol{X}_{w}^{n}+\int_{t^{n}}^{t^{n+1}} \boldsymbol{V}(t) \mathrm{d} t
$$

The vector $\boldsymbol{V}$ in this equation includes the local (inertial) flow velocities at the grid points of the wake mesh. If a prescribed wake were to be considered, the integral term would be dropped, but for a fully force-free wake it is necessary to retain it. $C_{b}$ and $C_{w}$ in Eq. (4) are very sparse constant matrices that update the position of the prescribed wake: the former closes the newly shed wake panel with the trailing edge of the lifting surface, satisfying the Kutta condition, while the latter preserves the wake of the previous time step unchanged.

For a free wake, the vortex-ring cornerpoints need also to be moved according to the local velocity, $V$, which encompasses bound and wake vorticity influence. As shown in Eq. (4), time-integration is necessary to determine the location of the rolled-up wake. Conventionally, this is done using an explicit one-step Euler 
method. In order to improve the accuracy and/or stability of the wake rollup, other higher-order schemes have been proposed in the literature, such as a two-step Euler ${ }^{15}$ and the fourth order Adams-BashforthMoulton. ${ }^{16}$ In this work it will be approximated as

$$
\int_{t^{n}}^{t^{n+1}} \boldsymbol{V}(t) \mathrm{d} t \approx\left[D_{b} \boldsymbol{\Gamma}_{b}^{n}+D_{w} \boldsymbol{\Gamma}_{w}^{n}\right] \Delta t
$$

where $D_{b}=D_{b}\left(\boldsymbol{X}_{b}^{n+\epsilon}, \boldsymbol{X}_{w}^{n+\epsilon}\right)$ and $D_{w}=D_{w}\left(\boldsymbol{X}_{w}^{n+\epsilon}\right)$ are analogous to $A_{b}$ and $A_{w}$ in Eq. (2), but with the induced velocities computed at the wake cornerpoints, instead of the bound collocation points.

In turn, the propagation equations for the wake circulation can be written in discrete time as

$$
\boldsymbol{\Gamma}_{w}^{n+1}=B_{b} \boldsymbol{\Gamma}_{b}^{n}+B_{w} \boldsymbol{\Gamma}_{w}^{n},
$$

where $B_{b}$ and $B_{w}$ are very sparse constant matrices which account for Kelvin's circulation theorem (that enforces the condition for wake shedding at the trailing edge) and Helmholtz's vortex theorem (in the convection of the wake). They serve a similar purpose as $C_{b}$ and $C_{w}$ in Eq. (4), mapping the wake circulation of the previous time step to the current one. Note that if any dissipation model were to be assumed for the wake, this should be included in $B_{w}$.

Finally, once the distribution of vortex elements has been obtained at each time step, the inviscid aerodynamic loads can be computed. The induced drag, $D$, is aligned with the local instantaneous velocity, and the lift, $L$, acts along the local vector perpendicular to the local velocity and projected over the normal to the panel. These loads are given by

$$
\begin{aligned}
\boldsymbol{L}^{n} & =\rho_{\infty} G_{c}\left[\left(U_{i} \Delta_{i}+U_{j} \Delta_{j}\right) \boldsymbol{\Gamma}_{b}^{n}+\dot{\boldsymbol{\Gamma}}_{b}^{n}\right], \\
\boldsymbol{D}^{n} & =\rho_{\infty}\left[-U^{*} \Delta_{i} \boldsymbol{\Gamma}_{b}^{n}+G_{s} \dot{\boldsymbol{\Gamma}}_{b}^{n}\right],
\end{aligned}
$$

where $\Delta_{i(j)}$ are matrices filled with 1 and -1 in the correct positions in order to account for adjacent panels (see Katz and Plotkin ${ }^{10}$ ); matrix $G_{c}=G_{c}\left(\boldsymbol{X}_{b}^{n}\right)$ and $G_{s}=G_{s}\left(\boldsymbol{X}_{b}^{n}\right)$ are diagonal matrices, with element $(k, k)$ given by $\left(G_{c}\right)_{k, k}=\left(\Delta b \Delta c \cos \alpha^{n}\right)_{k},\left(G_{s}\right)_{k, k}=\left(\Delta b \Delta c \sin \alpha^{n}\right)_{k}$, and $\alpha_{k}^{n}\left(\boldsymbol{X}_{b}^{n}, \dot{\boldsymbol{X}}_{b}^{n}\right)$ represents the angle of incidence of vortex ring $k$ at time step $n ; U_{i(j)}=U_{i(j)}\left(\boldsymbol{\Gamma}_{w}^{n}, \boldsymbol{X}_{b}^{n}, \boldsymbol{X}_{w}^{n}, \dot{\boldsymbol{X}}_{b}^{n}\right)$ and $U^{*}=U^{*}\left(\boldsymbol{\Gamma}_{b}^{n}, \boldsymbol{\Gamma}_{w}^{n}, \boldsymbol{X}_{b}^{n}, \boldsymbol{X}_{w}^{n}\right)$ are diagonal matrices that store the induced velocities projected over the relevant vectors and weighted using the corresponding geometric properties, such that

$$
\left(U_{i}\right)_{k, k}=\left(\frac{\vec{q} \cdot \vec{\tau}_{i}}{\Delta c}\right)_{k}, \quad\left(U_{j}\right)_{k, k}=\left(\frac{\vec{q} \cdot \vec{\tau}_{j}}{\Delta b}\right)_{k}, \quad \text { and } \quad\left(U_{j}\right)_{k, k}^{*}=\left(\vec{q}^{*} \cdot \vec{l} \Delta b\right)_{k} .
$$

The induced velocities $\vec{q}$ include contributions from the wake and the motion of the lifting surface, whereas $\vec{q}^{*}$ only considers the streamwise contribution of bound and wake circulation (plus the trailing-edge closing segment); $\vec{\tau}_{i}, \vec{\tau}_{j}$ are, respectively, the chordwise and spanwise tangential vectors of the panel, and $\vec{l}$ represents the local lift vector; $\Delta c$ and $\Delta b$ correspond to the chordwise and spanwise panel dimensions.

To sum up, equations (2), (4) and (6) define the discrete-time propagation equations in the aerodynamic states defined in Eq. (1). The expressions for the aerodynamic loads, Eqs. (7-8) are the output equations. Note that the derivative of the bound circulation, $\dot{\boldsymbol{\Gamma}}_{b}$, appears in the evaluation of the unsteady lift and induced drag, and therefore it was included in the state vector. An alternative procedure would have been to substitute directly $\dot{\boldsymbol{\Gamma}}_{b}^{n}$ in Eqs. (7-8), obtained from the non-penetration boundary condition, Eq. (2), together with Eqs. (3-6), in which case this term would not need to be kept explicitly. The current approach was found however more appropriate since it is easier to implement and the penalty in the number of states is not significant.

\section{Flexible Body Dynamics: Displacement-Based Geometrically-Exact Composite Beam}

The slender structures in the high-aspect-ratio wing aircraft will be modeled as composite beams, using a finite-element solution methodology based on those of Hodges ${ }^{17}$ and Patil et al., ${ }^{2}$ but using displacements and the Cartesian Rotation Vector (CRV) as primary degrees of freedom, as done by Geradin and Cardona. ${ }^{18} \mathrm{~A}$ quick summary of the solution procedure is included here for completeness. Figure 2 sketches the description 
of the deformation that will be followed here. There are no constraints on the undeformed configuration allowing the beam to be initially curved and twisted.

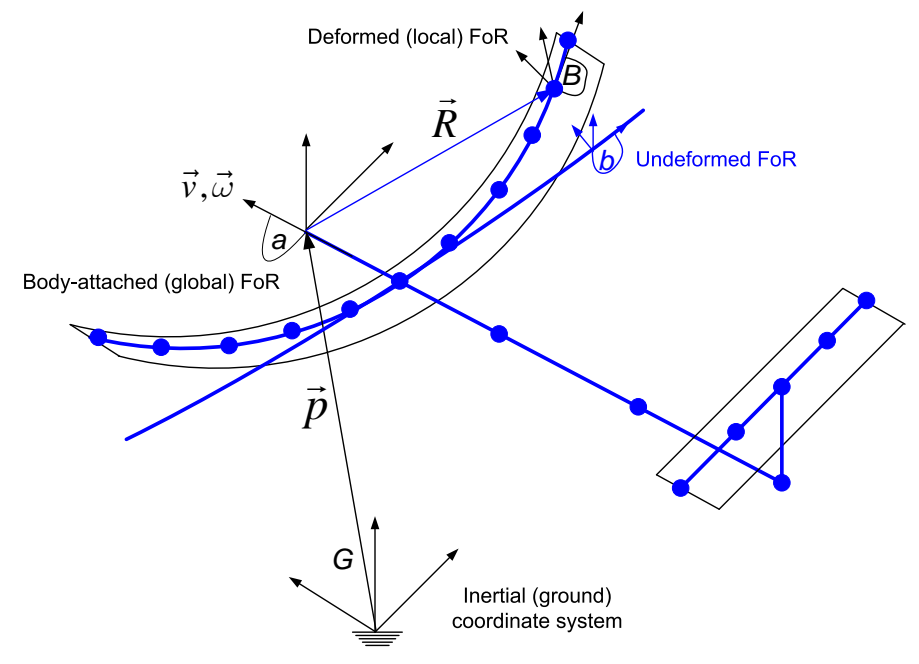

Figure 2. Structural model: geometrically-exact beam elements.

\section{A. Geometrically-Exact Beam Kinematics using the Cartesian Rotation Vector}

The deformation of the structure is described in a Lagrangian manner in terms of a moving, body-fixed reference coordinate system $a$ which moves with respect to an inertial frame $G$ by the translational velocity of its origin $v_{a}(t)$ and its rotational velocity $\omega_{a}(t)$. Subscripts are used to indicate the coordinate system in which the components of the vectors are given. The orientation of the global frame $a$ with respect to the inertial frame $G$ is given by the coordinate transformation matrix, $C^{G a}(t)$ and will be parameterized using quaternions $\zeta(t)=\left(\zeta_{0}, \zeta_{v}\right)$.

The local orientation of the beam cross sections is defined by their local coordinate systems, $B$, in the deformed (or current) configuration. The orientation of cross-sections at each point in the current configuration is described in terms of finite rotations from the global reference frame $a$ and the local deformed frame $B$ using the CRV, $\Psi(s, t)$. The corresponding coordinate transformation matrix will be $C^{B a}(s, t)$. The deformation of the reference line going from the undeformed state $\left\{\vec{R}(s, 0), \vec{B}_{i}(s, 0)\right\}$ to the current state $\left\{\vec{R}(s, t), \vec{B}_{i}(s, t)\right\}$ will be described by the following force and moment strains ${ }^{17}$

$$
\begin{aligned}
\gamma(s, t) & =C^{B a}(s, t) R_{a}^{\prime}(s, t)-C^{B a}(s, 0) R_{a}^{\prime}(s, 0), \\
\kappa(s, t) & =K_{B}(s, t)-K_{B}(s, 0),
\end{aligned}
$$

where $(\bullet)^{\prime}$ is the derivative with respect to the arclength $s$. The curvature will be computed from the corresponding CRV, $\Psi$ for the rotation from frame $a$ to frame $B$,

$$
K_{B}=T(\Psi) \Psi^{\prime}
$$

where $T(\Psi)$ is the tangential operator, which can be written as, ${ }^{18}$

$$
T(\Psi)=I+\frac{\cos \phi-1}{\phi^{2}} \tilde{\Psi}+\left(1-\frac{\sin \phi}{\phi}\right) \frac{\tilde{\Psi} \tilde{\Psi}}{\phi^{2}},
$$

where $(\tilde{\bullet})$ is the cross-product operator and $\phi=\|\Psi\|$. The inertial properties of the reference line will be determined by its translational and angular inertial velocities at each location defined by the arclength $s$, given, respectively, as

$$
\begin{aligned}
V_{B} & =C^{B a}\left(\dot{R}_{a}+\tilde{\omega}_{a} R_{a}+v_{a}\right), \\
\Omega_{B} & =T(\Psi) \dot{\Psi}+C^{B a} \omega_{a} .
\end{aligned}
$$




\section{B. Dynamic Equations of Motion for an Unrestrained Curved Beam}

The dynamics of the beam in a given time interval $\left[t_{1}, t_{2}\right]$ is to be analyzed in the (moving) body-attached reference frame $a$. From Hamilton's principle, it is

$$
\int_{t_{1}}^{t_{2}} \delta \Pi d t=\int_{t_{1}}^{t_{2}} \int_{0}^{l}[\delta \mathcal{T}-\delta \mathcal{U}+\delta \mathcal{W}] d s d t=0 .
$$

In this expression, $\Pi$ is defined as the total potential of the member, $\mathcal{T}$ and $\mathcal{U}$ are the kinetic and internal energy densities per unit length, respectively, and $\delta \mathcal{W}$ is the virtual work of applied loads per unit length. The virtual strain and kinetic energies are written, respectively, as

$$
\begin{gathered}
\delta \mathcal{U}=\delta \gamma^{T} F_{B}+\delta \kappa^{T} M_{B}, \\
\delta \mathcal{T}=\delta V_{B}^{T} P_{B}+\delta \Omega_{B}^{T} H_{B},
\end{gathered}
$$

where $F_{B}$ and $M_{B}$ are the column matrices with the components of the internal force and moment in the local deformed frame. They are related to the beam strains through the constitutive relations

$$
\left\{\begin{array}{c}
F_{B} \\
M_{B}
\end{array}\right\}=[\mathcal{S}] \cdot\left\{\begin{array}{l}
\gamma \\
\kappa
\end{array}\right\} .
$$

The stiffness matrix $\mathcal{S}$ is obtained through an appropriate cross-sectional analysis methodology. ${ }^{19}$ The inertial velocities in Eq. (17) are also given in their components in the material frame $B$. Their conjugated momenta are defined then as

$$
\left\{\begin{array}{l}
P_{B} \\
H_{B}
\end{array}\right\}=[\mathcal{M}] \cdot\left\{\begin{array}{l}
V_{B} \\
\Omega_{B}
\end{array}\right\},
$$

where the cross-sectional mass matrix $\mathcal{M}$ is given as

$$
\mathcal{M}=\left[\begin{array}{cc}
m I & -m \tilde{\xi}_{B}^{c g} \\
m \tilde{\xi}_{B}^{c g} & J
\end{array}\right],
$$

with mass per unit length $m$, cross-sectional inertia, $J$, and distance between the reference line of the beam and the material centroid of the cross sections, $\vec{\xi}^{c g}$. Finally, all virtual magnitudes will be expressed in terms of the independent set of variables, through the relations introduced in the previous section.

To obtain the virtual work of the applied forces in Eq. (15), consider the surface forces $\vec{\mu}$ (given in its components $\mu_{G}$ in the inertial frame of reference) acting on the external contour of the beam cross sections. The corresponding virtual work per unit length is given by

$$
\delta \mathcal{W}=\left\langle\delta X_{G}^{T} \mu_{G}\right\rangle
$$

where $\delta X_{G}$ is the virtual position vector in the current configuration at the material points where $\vec{\mu}$ is applied and $\langle\bullet\rangle$ is the integral over the area contour at the cross section at position $s$ along the reference line. From Figure 2, the position vector itself is

$$
X_{G}=p_{G}+C^{G a} R_{a}+C^{G a} C^{a B} \xi_{B}
$$

where the column matrix $\xi_{B}$ contains the cross-sectional coordinates expressed in the local deformed material frame $B$. Substituting this expression in Eq. (21), one obtains the following form for the virtual work per unit length

$$
\delta \mathcal{W}=\left[\delta p_{G}^{T} C^{G a}+\delta R_{a}^{T}+\delta \phi_{a}^{T}\left(\tilde{R}_{a}+C^{a B} \tilde{\xi}_{B} C^{B a}\right)\right] C^{a B} f_{B}+\delta \Phi_{B}^{T} m_{B},
$$

where $\delta \phi_{a}$ and $\delta \Phi_{B}$ are global and local virtual rotations, respectively, and where the set of resultant forces per unit length have been obtained as

$$
f_{B}=\left\langle\mu_{B}\right\rangle, m_{B}=\left\langle\tilde{\xi}_{B} \mu_{B}\right\rangle .
$$


All the different contributions of the internal, kinetic and virtual work of the applied forces are substituted into the expression of Hamilton's principle Eq. (15). After integration by parts, the weak form of the equations of motion is

$$
\begin{aligned}
\int_{t_{1}}^{t_{2}} \int_{0}^{l} \delta & R_{a}^{T} C^{a B}\left[\left(\frac{d}{d t}+\tilde{\Omega}_{B}\right) P_{B}-f_{B}\right]+\delta R_{a}^{\prime T} C^{a B} F_{B}+ \\
& \delta \Phi_{B}^{T}\left[\left(\frac{d}{d t}+\tilde{\Omega}_{B}\right) H_{B}+\tilde{V}_{B} P_{B}-\tilde{K}_{B} M_{B}-\left(\tilde{e}_{1}+\tilde{\gamma}\right) F_{B}-m_{B}\right]+\delta{\Phi^{\prime}}_{B}^{T} M_{B} d s+ \\
& \delta p_{G}^{T} C^{G a}\left[\left(\frac{d}{d t}+\tilde{\omega}_{a}\right) \mathbb{P}_{a}-\mathbb{F}_{a}\right]+\delta \phi_{a}^{T}\left[\left(\frac{d}{d t}+\tilde{\omega}_{a}\right) \mathbb{H}_{a}-\mathbb{M}_{a}\right] d t= \\
& =\int_{0}^{l}\left[\delta R_{a}^{T} C^{a B} P_{B}+\delta \Phi_{B}^{T} H_{B}\right]_{t_{1}}^{t_{2}} d s+\left[\delta p_{G}^{T} C^{G a} \mathbb{P}_{a}+\delta \phi_{a}^{T} \mathbb{H}_{a}\right]_{t_{1}}^{t_{2}}
\end{aligned}
$$

where $e_{1}=\left[\begin{array}{lll}1 & 0 & 0\end{array}\right]^{T}$. The total momenta and external forces are given in the global reference frame $a$ as

$$
\begin{array}{ll}
\mathbb{P}_{a}=\int_{0}^{l} C^{a B} P_{B} d s, & \mathbb{H}_{a}=\int_{0}^{l}\left(\tilde{R}_{a} C^{a B} P_{B}+C^{a B} H_{B}\right) d s \\
\mathbb{F}_{a}=\int_{0}^{l} C^{a B} f_{B} d s, & \mathbb{M}_{a}=\int_{0}^{l}\left(\tilde{R}_{a} C^{a B} f_{B}+C^{a B} m_{B}\right) d s .
\end{array}
$$

In strong form, the flexible-body dynamics equations can be written as

$$
\begin{aligned}
& \left(\frac{d}{d t}+\tilde{\Omega}_{B}\right) P_{B}=\left(\frac{d}{d x}+\tilde{K}_{B}\right) F_{B}+f_{B}, \\
& \left(\frac{d}{d t}+\tilde{\Omega}_{B}\right) H_{B}+\tilde{V}_{B} P_{B}=\left(\frac{d}{d x}+\tilde{K}_{B}\right) M_{B}+\left(\tilde{e}_{1}+\tilde{\gamma}\right) F_{B}+m_{B}, \\
& \left(\frac{d}{d t}+\tilde{\omega}_{a}\right) \mathbb{P}_{a}=\mathbb{F}_{a}, \\
& \left(\frac{d}{d t}+\tilde{\omega}_{a}\right) \mathbb{H}_{a}=\mathbb{M}_{a} .
\end{aligned}
$$

The orientation of the body-fixed reference frame with respect to the inertial frame will be represented by means of quaternions, which need to satisfy the propagation equation ${ }^{20}$

$$
\begin{aligned}
& \dot{\zeta}_{0}=-\frac{1}{2} \omega_{a}^{T} \zeta_{v}, \\
& \dot{\zeta}_{v}=\frac{1}{2}\left(\zeta_{0} \omega_{a}-\tilde{\omega}_{a} \zeta_{v}\right) .
\end{aligned}
$$

The instantaneous coordinate transformation matrix $C^{G a}$ and position vector of the body-fixed reference frame are finally obtained as

$$
\begin{aligned}
C^{G a} & =\left(2 \zeta_{0}^{2}-1\right) I+2\left(\zeta_{v} \zeta_{v}^{T}+\zeta_{0} \tilde{\zeta}_{v}\right), \\
\dot{p}_{G} & =C^{G a} v_{a} .
\end{aligned}
$$

\section{Discrete Form of the Equations of Motion}

Eq. (25) sets the basis for a finite-element discretization solution procedure. For that purpose, we will approximate the position and rotation vectors within the $n^{\text {th }}$ element by given shape functions $N_{i}(s)^{\text {a }}$,

$$
\begin{aligned}
R_{a}(s) & \cong \sum_{i=1}^{3} N_{i}(s) R_{a}\left(s_{i}\right), \\
\Psi(s) & \cong \sum_{i=1}^{3} N_{i}(s) \Psi\left(s_{i}\right),
\end{aligned}
$$

\footnotetext{
aThere are known issues with objectivity of the interpolation operation of finite rotations. ${ }^{21}$ At the time of this manuscript this is still under investigation, but good performance of the implementation has been observed for fine enough discretization.
} 
for $s_{n-1} \leq s \leq s_{n}$. Linear and quadratic elements have been implemented. If $\boldsymbol{\eta}$ is the vector of all nodal displacements and rotations, the discrete form of the dynamic equations is written as

$$
M(\boldsymbol{\eta})\left\{\begin{array}{c}
\ddot{\boldsymbol{\eta}} \\
\dot{v}_{a} \\
\dot{\omega}_{a}
\end{array}\right\}+Q_{g y r}\left(\boldsymbol{\eta}, \dot{\boldsymbol{\eta}}, v_{a}, \omega_{a}\right)+Q_{s t i f}(\boldsymbol{\eta})=Q_{e x t}\left(\boldsymbol{\eta}, \dot{\boldsymbol{\eta}}, v_{a}, \omega_{a}, \zeta\right)
$$

Details of the different terms in this equation are included in the appendix. Finally, the linearized (incremental) form of Eq. (31) around a dynamic equilibrium is given as

$$
M\left\{\begin{array}{c}
\Delta \ddot{\boldsymbol{\eta}} \\
\Delta \dot{v}_{a} \\
\Delta \dot{\omega}_{a}
\end{array}\right\}+C\left\{\begin{array}{c}
\Delta \dot{\boldsymbol{\eta}} \\
\Delta v_{a} \\
\Delta \omega_{a}
\end{array}\right\}+K\left\{\begin{array}{c}
\Delta \boldsymbol{\eta} \\
0 \\
0
\end{array}\right\}=\Delta Q_{e x t}\left(\Delta \boldsymbol{\eta}, \Delta \dot{\boldsymbol{\eta}}, \Delta v_{a}, \Delta \omega_{a}, \Delta \zeta\right)
$$

where $M, C$, and $K$ are the tangent mass, damping and stiffness matrices.

\section{Coupled Aeroelasticity and Flight Dynamics of a Flexible Aircraft}

The previous flexible-body and unsteady aerodynamic models will be used to represent the complete dynamics of a flexible air vehicle. As the structural model is based on beams (curves in space) and the aerodynamic lattice is distributed over a lifting surface (see Figure 3), a mapping procedure is required between both meshes.

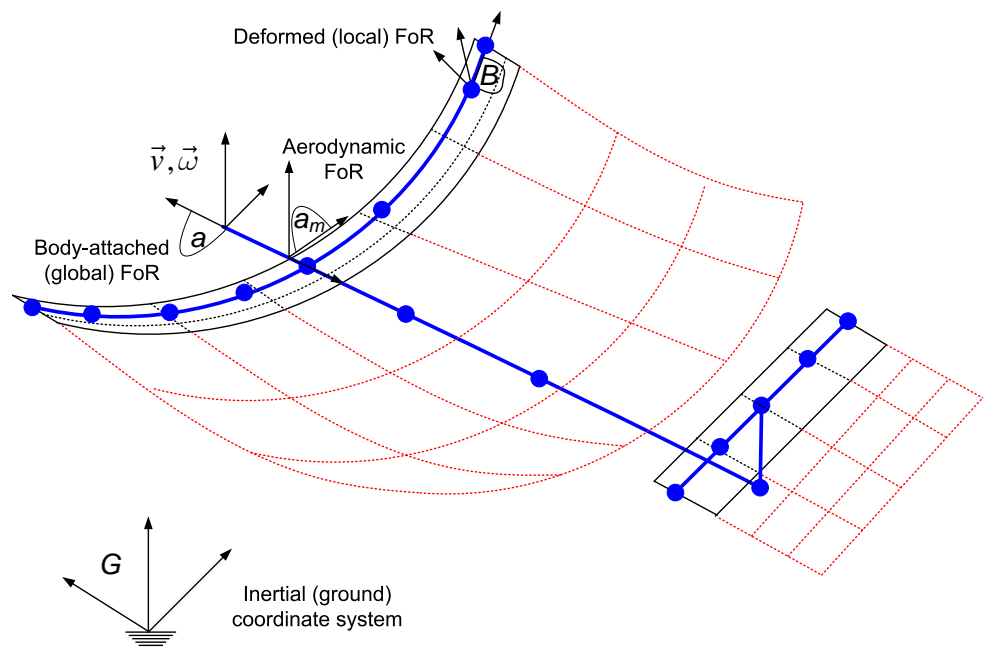

Figure 3. Full representation of flexible aircraft: beam-like structure, vortex-ring lattice and rigid-body motions.

\section{A. Mapping Structural Displacement to the Aerodynamic Model}

Firstly, displacements and rotations of the beam nodes, $\boldsymbol{R}_{a}$ and $\boldsymbol{\Psi}$, and the corresponding velocities, $\dot{\boldsymbol{R}}_{a}$ and $\dot{\boldsymbol{\Psi}}$, have to be transformed to deformations and velocities of the grid points of the aerodynamic lattice, which

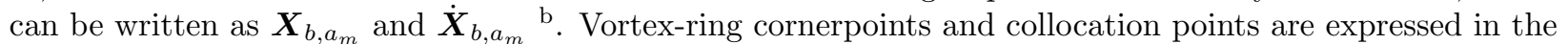
aerodynamic coordinate system, $a_{m}$, defined independently but rigidly linked to the body-fixed global one, $a$, and can be mapped to and from the other reference frames through transformation matrices. It would be possible to include camber deformations on this approach, ${ }^{22}$ but it will be assumed here that airfoils remain rigid under wing deformations.

\footnotetext{
b In what follows, subindex $b$ will be dropped from these magnitudes, since only the lifting surfaces are considered in the interface with the structural model. We will refer to these magnitudes as $\boldsymbol{X}_{a_{m}}$ and $\dot{\boldsymbol{X}}_{a_{m}}$ to indicate that they are referred to the aerodynamic frame of reference of the $m$ lifting surface.
} 


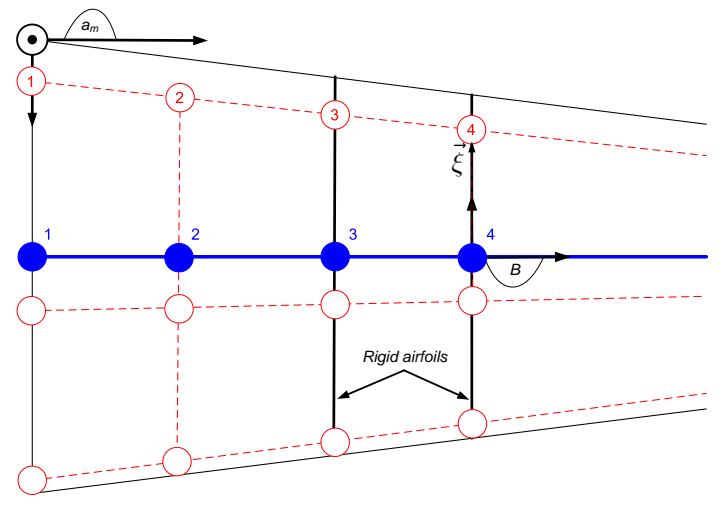

(a) Mapping between beam nodes and aerodynamic grid. A discontinuous line is used for the vortex rings and a continuous line for the beam. Aerodynamic cornerpoints are hollow, whereas beam nodes are filled.

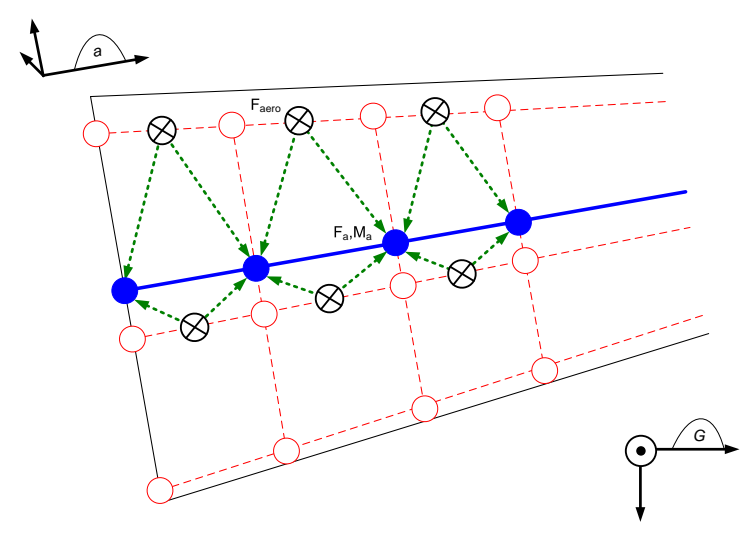

(b) Mapping of inviscid aerodynamic forces, $F_{a e r o}$, to nodal forces and moments $\left(F_{a}, M_{a}\right)$. Locations at which aerodynamic forces act (center of vortex ring leading segment) are marked with crosses.

Figure 4. Mapping between aerodynamic lattice and structural finite element discretization: (a) geometry and (b) aerodynamic loads.

In the initial configuration a mapping between the structural nodes and the aerodynamic grid can be defined, as illustrated in Figure 4(a). For the sake of simplicity, the finite-element discretization of the beam coincides with the spanwise aerodynamic grid, and hence vortex ring cornerpoints and beam nodes lie along the same rigid airfoil at each spanwise station - note, however, that cambered airfoils are allowed and that non-coinciding meshes have also been implemented. The variable $\vec{\xi}$ measures the distance between a vortex ring cornerpoint and the relevant node, and it will be expressed in the nodal material frame, i.e., $\xi_{B}$. This quantity will remain constant under the assumption of rigid airfoils, and as a consequence, it is possible to determine the aerodynamic grid in the lifting surface aerodynamic frame of reference, $a_{m}$, at any deformed configuration of the member. For a given vortex ring cornerpoint, the following transformation is defined at time step $n$ :

$$
X_{a_{m}}^{n}=C^{a_{m} a}\left[R_{a}^{n}+C^{a B}\left(\Psi^{n}\right) \xi_{B}\right],
$$

where the coordinate transformation between the body-fixed global coordinate system, $a$, and the aerodynamic frame, $a_{m}$, is given by the constant $C^{a_{m} a}$ matrix. Each cornerpoint of the vortex rings is updated analogously. In turn, the positions of the collocation points are obtained through interpolation of the corresponding four vortex ring cornerpoints.

The transformation for the velocities is

$$
\dot{X}_{a_{m}}^{n}=C^{a_{m} a}\left[v_{a}^{n}+\widetilde{\omega}_{a}^{n} R_{a}^{n}+\dot{R}_{a}^{n}+C^{a B}\left(\Psi^{n}\right) \widetilde{\Omega}_{B}^{n} \xi_{B}\right],
$$

where the local inertial angular velocity, $\Omega_{B}^{n}$, was given in Eq. (14). As in the case of positions, the velocities of the aerodynamic vortex ring cornerpoints are obtained using Eq. (34), and the velocities of the collocation points are obtained through interpolation.

\section{B. Mapping Aerodynamic Forces to the Structural Model}

Secondly, it is necessary to transform the inviscid aerodynamic loads computed in Eqs. (7-8) to forces and moments acting upon the beam nodes - any estimation of the viscous drag will be included in the inputs to the system. For that purpose, it is assumed that they can be approximated by isolated aerodynamic loads applied in the center of the leading segment of each vortex-ring.

The aerodynamics forces act on the plane defined by the instantaneous inertial velocity of the vortex ring (computed at the collocation point), and the normal vector of the vortex ring (expressed in the inertial frame, $G)$. The pressure differential acts along the normal vector, but due to the inability of the UVLM to 
account for the leading edge suction, only the component normal to the inertial velocity is considered. In turn, the induced drag acts along the vector defined by the local instantaneous velocity. As a result, the inviscid aerodynamic forces at vortex ring $k$, expressed in the three axis defined by the inertial (ground) frame of reference, $G$, are given by

$$
\left(F_{\text {aero }}^{n}\right)_{k}=C^{G A}\left\{\begin{array}{l}
D \\
0 \\
L
\end{array}\right\}_{k}^{n}
$$

with $L^{n}$ and $D^{n}$ the lift and the induced drag of the panel, as given by Eqs. (7-8); $C^{G A}=C^{G A}\left(p_{a}^{n}, \zeta^{n}, \dot{\boldsymbol{X}}_{b}^{n}\right)$ is the coordinate transformation matrix between the ground frame and the local aerodynamic frame linked to the wing airfoils (determined by the local instantaneous inertial velocity and the normal vector the wing).

These forces are then lumped into the nodes of the deformed beam, splitting them between adjacent nodes as illustrated in Figure 4(b) - note that this mapping will give rise to moments acting upon the corresponding nodes. Once the resulting nodal forces and moments have been computed, $\left(F_{G}, M_{G}\right)$, they are transformed to the body-fixed $a$ frame, in order to be consistent with the flexible-beam equations, Eqs. (28) and (31). These operations can be summarized as

$$
\left\{\begin{array}{c}
\boldsymbol{F}_{a} \\
\boldsymbol{M}_{a}
\end{array}\right\}^{n}=\bar{C}^{a G}\left\{\begin{array}{c}
\boldsymbol{F}_{G} \\
\boldsymbol{M}_{G}
\end{array}\right\}^{n}=\bar{C}^{a G} \chi_{v r \rightarrow n o} \boldsymbol{F}_{\text {aero }}^{n}
$$

where $\bar{C}^{a G}$ is a block diagonal matrix, being each block given by the corresponding coordinate transformation matrix from the inertial to the body-fixed frame, $C^{a G}=C^{a G}\left(\zeta^{n}\right) ; \chi_{v r \rightarrow n o}=\chi_{v r \rightarrow n o}\left(\boldsymbol{R}_{a}^{n}, \Psi^{n}, \boldsymbol{X}_{b}^{n}\right)$ is a very sparse matrix that lumps the forces acting on the aerodynamic lattice vortex rings, $\boldsymbol{F}_{\text {aero }}^{n}=$ $\boldsymbol{F}_{\text {aero }}\left(p_{a}^{n}, \zeta^{n}, \boldsymbol{\Gamma}_{b}^{n}, \boldsymbol{\Gamma}_{w}^{n}, \boldsymbol{X}_{b}^{n}, \boldsymbol{X}_{w}^{n}, \dot{\boldsymbol{X}}_{b}^{n}\right)$, into forces and moments applied on the beam nodes expressed in the inertial frame, $\left(\boldsymbol{F}_{G}^{n}, \boldsymbol{M}_{G}^{n}\right)$.

The aerodynamic loads will also affect the rigid-body motions of the aircraft. The forces and moments acting at the origin of the body-fixed frame of reference, $\left(f_{a}, m_{a}\right)$, are obtained by integrating the nodal values and can be expressed as

$$
\left\{\begin{array}{c}
f_{a} \\
m_{a}
\end{array}\right\}^{n}=\chi_{n o \rightarrow b f}\left\{\begin{array}{c}
\boldsymbol{F}_{a} \\
\boldsymbol{M}_{a}
\end{array}\right\}^{n}=\chi_{n o \rightarrow b f} \bar{C}^{a G} \chi_{v r \rightarrow n o} \boldsymbol{F}_{\text {aero }}^{n}
$$

where $\chi_{n o \rightarrow b f}=\chi_{n o \rightarrow b f}\left(\boldsymbol{R}_{a}^{n}, \Psi^{n}\right)$ is the matrix that computes the resultant forces and moments integrating contributions of all nodes of the discretization. Hence, the generalized aerodynamic forces can be written as

$$
Q_{\text {aero }}^{n}=\left\{\begin{array}{c}
Q_{\text {aero }}^{S} \\
Q_{\text {aero }}^{R}
\end{array}\right\}^{n},
$$

with

$$
Q_{\text {aero }}^{S}=\left\{\begin{array}{c}
\boldsymbol{F}_{a} \\
\boldsymbol{M}_{a}
\end{array}\right\} \text { and } Q_{\text {aero }}^{R}=\chi_{n o \rightarrow b f}\left\{\begin{array}{c}
\boldsymbol{F}_{a} \\
\boldsymbol{M}_{a}
\end{array}\right\} .
$$

These generalized aerodynamic forces will be part of the generalized external forces presented in Eq. (31), which will also encompass any other applied loads, such that

$$
Q_{e x t}^{n}=Q_{a e r o}\left(p_{a}^{n}, \zeta^{n}, \boldsymbol{\eta}^{n}, \boldsymbol{\Gamma}_{b}^{n}, \boldsymbol{\Gamma}_{w}^{n}, \boldsymbol{X}_{b}^{n}, \boldsymbol{X}_{w}^{n}, \dot{\boldsymbol{X}}_{b}^{n}\right)+Q_{a p p}\left(\boldsymbol{u}^{n}\right)
$$

\section{Solution Methods}

Through the above mapping procedures, the coupling of aerodynamic and structural models for a full aeroelastic and flight dynamics characterization of the flexible vehicle can be tackled. The flexible body dynamics equations, Eqs. (28) and (31) are integrated with the unsteady aerodynamics, Eqs. (2-8). Other external forces, in particular, gravity forces, can also be introduced into the equations of motion. Different solution approaches have been defined: static aeroelastic, trim, stability analysis and dynamic aeroelastic, including linearized and geometrically-nonlinear solutions, when appropriate. In particular, for fully nonlinear 
time-domain simulations, the second order continuous-time flexible-body equations are discretized using the Newmark- $\beta$ method, and a loosely coupled approach solves them together with the discrete-time UVLM formulation.

The stability studies are carried out about a geometrically-nonlinear deformed configuration (typically, a trimmed aircraft condition). The procedure is similar to that of Hall, ${ }^{23}$ with a discrete-time linearized state-space formulation of the unsteady aerodynamics, tightly coupled with the linearized structural and rigid-body dynamics equations, Eq. (32), defining a single aeroelastic system matrix. The linearization of the aerodynamic equations, Eq. (2-8), is carried out under the following assumptions:

- The deformations around the deformed aircraft are small, and as a consequence, the non-penetration boundary condition can be enforced at the statically-deformed reference geometry.

- The aerodynamic forces will always by applied at the equilibrium configuration. This simplification effectively converts them into dead loads that only depend on velocities, but not on positions - note that the dependency with angle of attack is actually retained in the matrix $W_{b}$, Eq. (3).

- Wake rollup around the reference can be neglected since the effect is small unless the lift coefficient is large, but can be accounted for to accurately trim the aircraft. This assumption reduces the UVLM to a prescribed-wake method, and under this approximation it is not necessary to keep track of the wake shape after trim. The aerodynamic states that fully define the UVLM are only circulation strength distributions and the derivative of the bound circulation. Note that even though the wake is prescribed in this case, it does not need to be flat, and it will be shed from the deformed lifting surface.

Freezing the geometry around the reference configuration leads to a linearized discrete-time state-space system of equations of the form $x^{n+1}=A x^{n}$, and the stability of the system is determined by the eigenvalues of the system matrix $A$. As the wake shape is not retained, the states of the coupled problem are

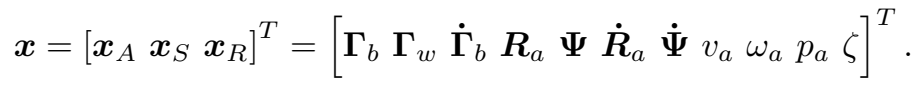

\section{Results}

The methods described above have been implemented in a new simulation framework, codenamed SHARP (Simulation of High Aspect-Ratio Planes). SHARP is built on a modular architecture in Matlab, but with low-level libraries in Fortran, that allows running independently the flexible-body dynamics and aerodynamics solvers, as well as the coupled system analysis described in Section IV.C. As it was also remarked above, the flexible-beam equations are geometrically exact, and therefore capture the nonlinearities that arise due to large deformations, updating the relevant inertia, gyroscopic and stiffness terms. Even though the vortex lattice method used for the unsteady aerodynamics is based on potential flow theory, the boundary conditions are enforced at the current deformed shape, thus accounting for the motion of the lifting surfaces. Aerodynamic control surfaces are directly modeled by prescribed motions of trailing edge panels and the true shape of the wake can be obtained as part of the solution procedure - however, a prescribed-wake model is often a good approximation and it has also been implemented. In a previous paper, ${ }^{7}$ we have presented static and dynamic validation of the structural dynamics solution under given loads, as well as unsteady aerodynamics with prescribed kinematics, and this will not be repeated here. This section includes verification studies on simple configurations, corresponding to a coupled structural dynamics/rigid-body dynamics problem, the dynamic aeroelastic stability of the Goland wing, and finally, on the open-loop response of two flexible vehicles.

\section{A. Flexible multibody dynamics solution}

The finite element analysis package SAMCEF is used first to verify the flexible-body dynamics implementation in SHARP. In particular, the SAMCEF module Mecano is a nonlinear dynamic analysis tool capable of solving flexible multibody problems and that follows the displacement-based description presented by Géradin and Cardona. ${ }^{18}$ The beam elements in SAMCEF cannot handle composite materials, and the results in this section are restricted to Aluminum beams.

Table 1 and Figure 5 show the geometry and material properties of the multibeam configuration for the analyzed problem. The flexible frame is subject to a pair of concentrated dead loads applied at the tips that 
Table 1. Simulation properties for the multibeam problem presented in Figure 5.

\begin{tabular}{ll}
\hline \hline Geometry: & $L_{1}=20 \mathrm{~m}$ and $L_{2}=5 \mathrm{~m}$ \\
& with $\mathrm{C}-\mathrm{S}=0.1 \mathrm{~m} \times 0.05 \mathrm{~m}$ solid \\
Material: & Aluminum \\
Elements: & 24 beam elements \\
Load: & $F_{0}=1000 \mathrm{~N}$ (non-follower) \\
Simulation: & $t_{0}=10 \mathrm{~s}$ and $d t=0.01 \mathrm{~s}$ \\
\hline \hline
\end{tabular}

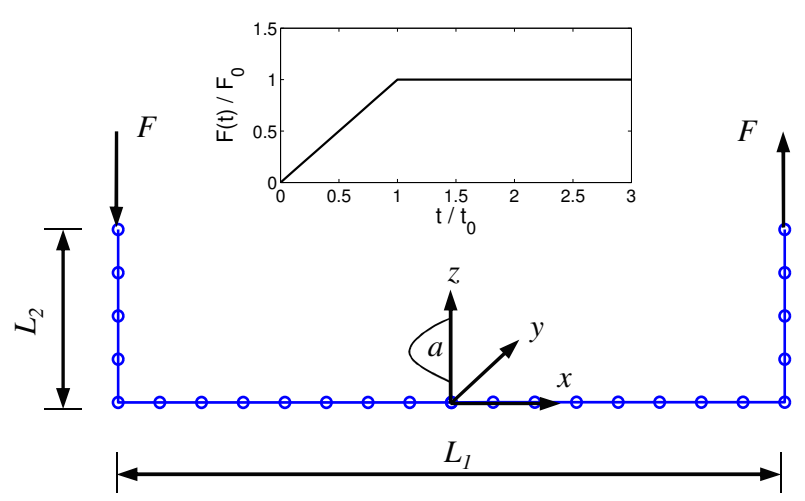

Figure 5. Geometry of multibeam configuration used for validation of SHARP flexible-body dynamics module. Simulation properties are presented in Table 1.

generate a resultant moment around the frame center of gravity. The frame is unsupported and there are no gravitational or aerodynamic forces. Both simulations, in SAMCEF and SHARP, correspond to a fixed time step size of $d t=0.01 \mathrm{~s}$ and use 24 linear elements.

The response of the structure over time is shown in Figure 6. To visualize the rigid-body motion and deformation of the frame, the deformed shape at 15 consecutive steps is shown in Figure 6 (a). It can be seen that the frame is undergoing large deformations which are perfectly captured in both simulations, as shown in Figure $6(\mathrm{~b})$. Figures 6 (c-d) show the velocities of the the body-fixed reference frame $a$ that is used to describe the rigid-body motion of the frame. All the variables in the figure are given in their components in an inertial frame.

\section{B. Flutter of the Goland wing using the state-space UVLM formulation}

The method for evaluation of dynamic stability outlined in section IV.C will be first exercised on the estimation of the flutter onset point of the Goland wing. ${ }^{24}$ This is a stiff cantilever wing for which Table 2 summarizes the relevant properties.

Table 2. Goland wing properties

\begin{tabular}{lrlr}
\hline \hline Chord, c & $1.8288 \mathrm{~m}$ & Mass per unit length & $35.71 \mathrm{~kg} / \mathrm{m}$ \\
Semi-span, B & $6.096 \mathrm{~m}$ & Moment of inertia (around e.a.) & $8.64 \mathrm{~kg} \cdot \mathrm{m}$ \\
Elastic axis (from l.e.) & $33 \%$ chord & Torsional stiffness & $0.99 \times 10^{6} \mathrm{~N} \cdot \mathrm{m}^{2}$ \\
Center of gravity (from l.e.) & $43 \%$ chord & Bending stiffness & $9.77 \times 10^{6} \mathrm{~N} \cdot \mathrm{m}^{2}$ \\
\hline \hline
\end{tabular}

Figure 7 presents the linear stability plot for the Goland wing. In this case, the flutter speed is computed around the undeformed configuration. For that purpose, a very small angle of attack of $\alpha=0.01 \mathrm{deg}$ has been prescribed in order create vorticity that can be shed into the wake. Air density is assumed to be $\rho_{\infty}=1.020 \mathrm{~kg} / \mathrm{m}^{3}$, which corresponds to an altitude of $1500 \mathrm{~m}$. The stability diagram indicates that the 

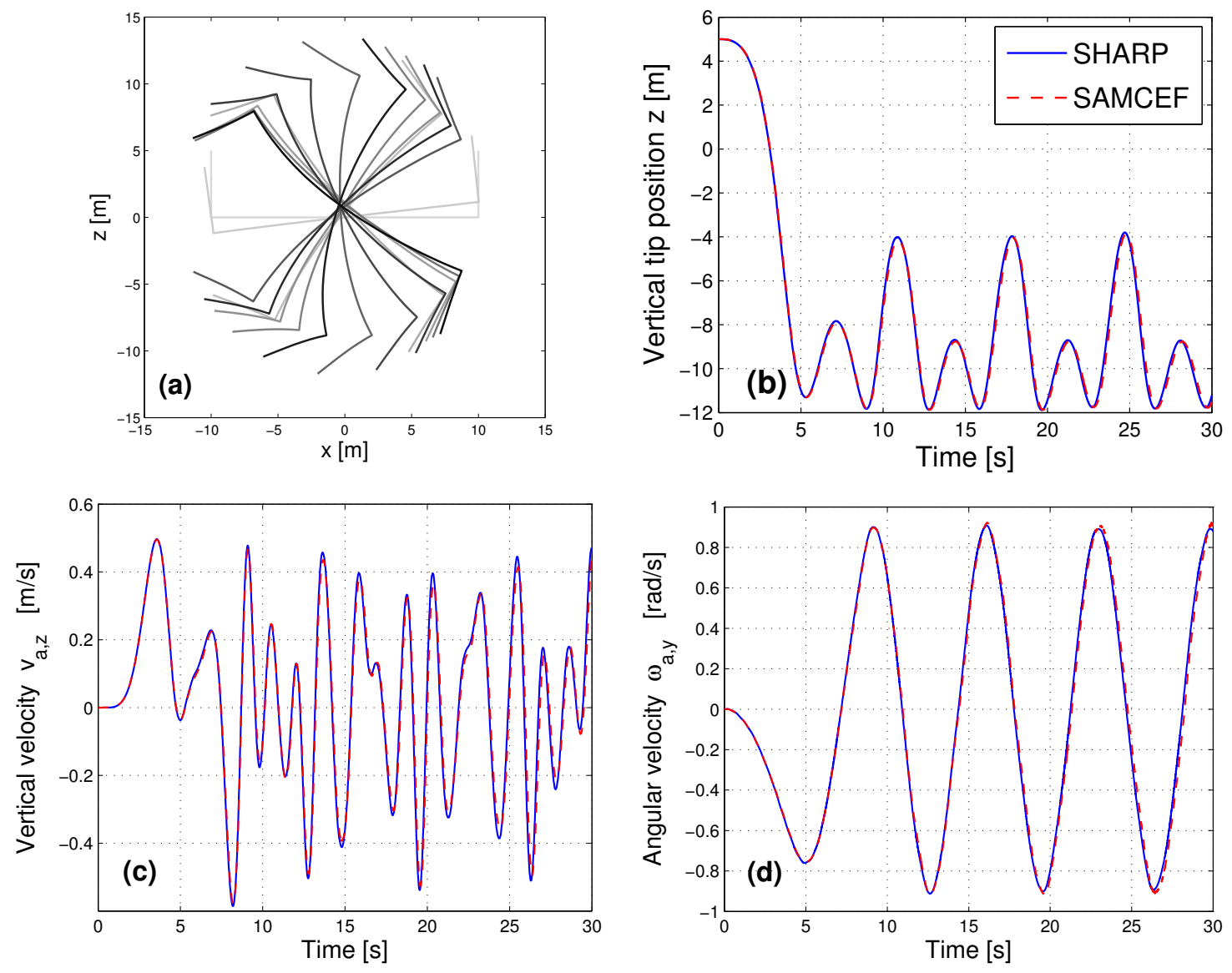

Figure 6. Response of the multibeam problem presented in Figure 5 subject to ramp dead loads applied at the tips. Time history of (a) the deformed shape at 15 consecutive steps from $t=0 \mathbf{s}$ (gray) to $t=30 \mathbf{s}$ (black), (b) vertical position $z$ of the left tip, (c) vertical linear velocity $v_{a, z}$ and (d) angular velocity $\omega_{a, y}$ of the body-fixed reference frame $a$ expressed in the inertial frame $G$.

first torsion mode becomes unstable at a velocity $V_{f}=166 \mathrm{~m} / \mathrm{s}$. The flutter speed of the Goland wing was also previously computed using SHARP both in time- and frequency-domain. ${ }^{9}$ Table 3 reproduces those results, those found in the literature, as well as those obtained with the present state-space stability analysis. The small discrepancies that can be observed are within the expected range.

It should be noted that very low free stream velocities were not included in Figure 7 . This is because the analysis must resolve in the small time steps required to capture the dominant structural modes of the wing and such study would lead to a prohibitively large number of wake circulation states in the state-space model. Note also that for flexible HALE vehicles the dominant structural modes will have far smaller frequencies, allowing for larger time steps and less wake states. This is is investigated next.

\section{Stability Studies on a Full Vehicle}

Flutter results are presented now on a full vehicle. The solution process is as follows: for a given velocity, the trimmed configuration of the free-flying vehicle is computed using the static aeroelastic solver. Then, a linear stability analysis is carried out. This is performed around the deformed configuration, unless otherwise stated - note that for a stable vehicle, this trimmed equilibrium may correspond to a highly deformed vehicle, or may even not exist at all. This matched solution has been dubbed nonlinear flutter solution in the literature. ${ }^{3}$

A numerical model of a flexible HALE vehicle has been defined (see Table 4 and Figure 8), loosely based on the one proposed by Patil and co-workers. ${ }^{3}$ The vehicle consists of a large aspect-ratio flexible wing, a rigid fuselage and and a rigid tail comprising a $25 \%$ chord elevator. The aircraft carries a payload of $50 \mathrm{~kg}$, located at $1 \mathrm{~m}$ from the elastic axis of the main wing, and is powered by two propellers, which are modeled 


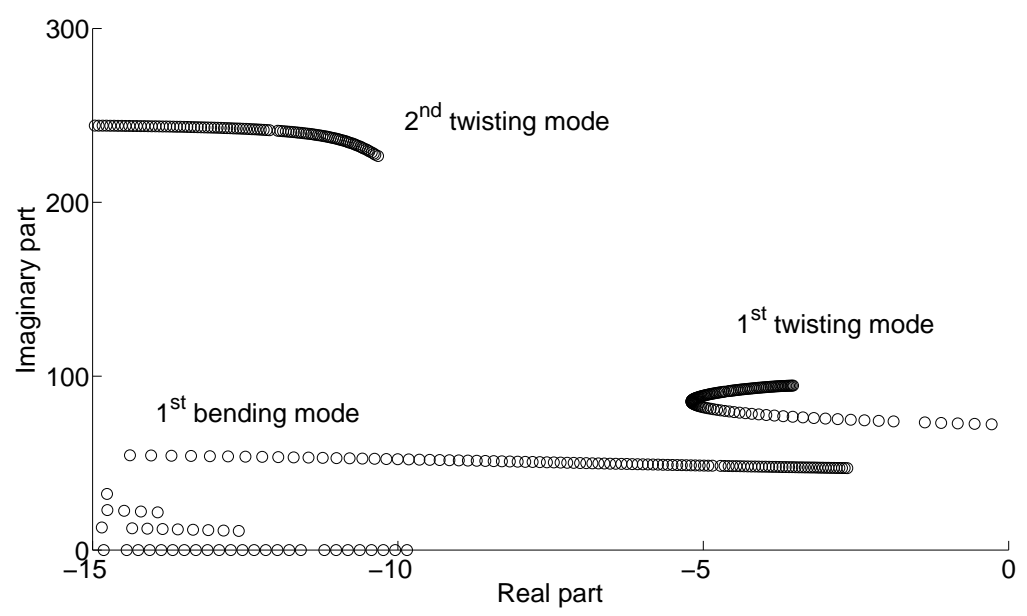

Figure 7. Stability plot for the undeformed Goland wing, computed with velocity increments of $\Delta V_{\infty}=1 \mathrm{~m} / \mathrm{s}$, starting at $V_{\infty}=35 \mathrm{~m} / \mathrm{s}$. Flutter occurs at $166 \mathrm{~m} / \mathrm{s} .\left[\rho_{\infty}=1.020 \mathrm{~kg} / \mathrm{m}^{3}\right.$ and $\left.\alpha=0.01 \mathrm{deg}\right]$

Table 3. Flutter speed of the Goland wing

\begin{tabular}{llcc}
\hline \hline Author & Model & $V_{f}, \mathrm{~m} / \mathrm{s}$ & $\omega_{f}, \mathrm{rad} / \mathrm{s}$ \\
\hline Goland $^{24}$ & Analytical & 172.1 & 67.4 \\
${\text { Wang et } \mathrm{al}^{4}}^{4}$ & ZAERO $^{25}$ & 174.3 & - \\
Wang et al $^{4}$ & Intrinsic beam + UVLM $^{\text {SHARP time-domain }}{ }^{9}$ & 163.8 & - \\
SHARP RFA & Displacement beam + UVLM & 165 & 69 \\
SHARP state-space stability & Displacement beam +UVLM & 177 & 68 \\
\hline \hline
\end{tabular}

as point forces rigidly linked to the wing. The present results only consider gravity forces for the payload. As observed in Table 4, the stiffness properties of the main wing will be used as a parameter for subsequent results, varying the parameter $\sigma$. In this case, it will be assumed that the aircraft flies at an altitude of 20 $\mathrm{km}$, where the density is $\rho_{\infty}=0.0899 \mathrm{~kg} / \mathrm{m}^{3}$.

The flutter speed has been computed for three cases: 1) the undeformed aircraft; 2) trimmed aircraft with static linear solver; and 3) the trimmed aircraft with static nonlinear solver. In the first case the flutter speed is obtained by linearizing with respect to the undeformed aircraft and as in the Goland wing case, a small angle of attack of $\alpha=0.01 \mathrm{deg}$ is prescribed. In the last two cases, the stability analysis is performed around the deformed state. The trimmed condition is achieved through three inputs, namely angle of attack, AoA, elevator deflection, $\delta$, and thrust per propeller, $T$. For each free stream velocity, the vehicle is trimmed first (if possible), and then, the system matrix is computed for this given deformed configuration. If the system is stable, the velocity is increased and the new trim conditions are determined, analyzing stability for the new equilibrium. Following this procedure, the matched flutter speed is obtained.

The inputs required to reach the trimmed steady level flight are presented first in Figure 9 for varying stiffness properties of the main wing - note that increasing $\sigma$ corresponds to a more flexible wing. The nondimensional tip deflection of the main wing at the trimmed configuration is also included, where $B=16 \mathrm{~m}$ corresponds to the semi-span of the wing. As it can be seen, the results of the linear and nonlinear solutions are very close up to wing tip deflection of around $15 \%$ of the wing semi-span. From that point they depart but still follow similar trends. Near that point there is also a maximum in all inputs to the vehicle trim. This point corresponds to a minimum in the flutter speed, as it can be seen in Figure 10. Radically different initial guesses have been tested to try to find other branches on the trim conditions, but only the depicted solution has been identified. The actual reason for this local change of trend with increased flexibility is still under investigation. The wing tip deflection, on the contrary, increases almost linearly with the wing 
Table 4. HALE model aircraft properties

\begin{tabular}{lll}
\hline \hline & Main wing & Tail \\
\hline Chord, c & $1 \mathrm{~m}$ & $0.5 \mathrm{~m}$ \\
Semi-span, B & $16 \mathrm{~m}$ & $2.5 \mathrm{~m}$ \\
Elastic axis (from l.e.) & $50 \%$ chord & N/A \\
Center of gravity (from l.e.) & $50 \% \mathrm{chord}$ & $50 \%$ chord \\
Mass per unit length & $0.75 \mathrm{~kg} / \mathrm{m}$ & $0.08 \mathrm{~kg} / \mathrm{m}$ \\
Moment of inertia (around e.a.) & $0.1 \mathrm{~kg} \cdot \mathrm{m}$ & $0.01 \mathrm{~kg} \cdot \mathrm{m}$ \\
Torsional stiffness & $\frac{1}{\sigma} \times 10^{4} \mathrm{~N} \cdot \mathrm{m}^{2}$ & $\infty$ \\
Bending stiffness & $\frac{2}{\sigma} \times 10^{4} \mathrm{~N} \cdot \mathrm{m}^{2}$ & $\infty$ \\
\hline \hline
\end{tabular}
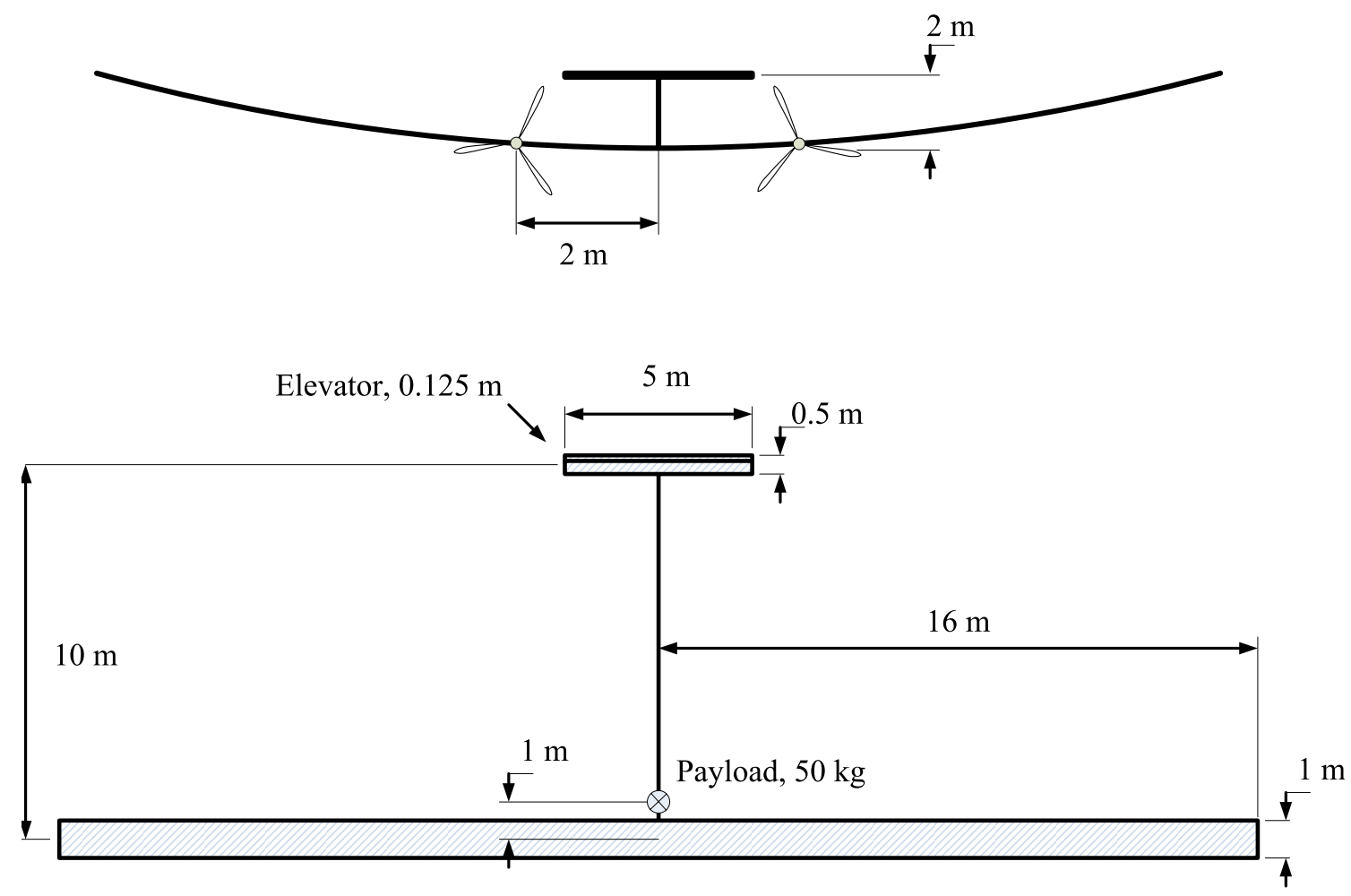

Figure 8. HALE model aircraft geometry. Large-aspect-ratio straight wing, rigid fuselage and T-tail, and propellers (not to scale). Front and top views, showing typical wing deformation. 
flexibility. This is because the total wing lift remains roughly constant across the different flight velocities to balance the constant weight.
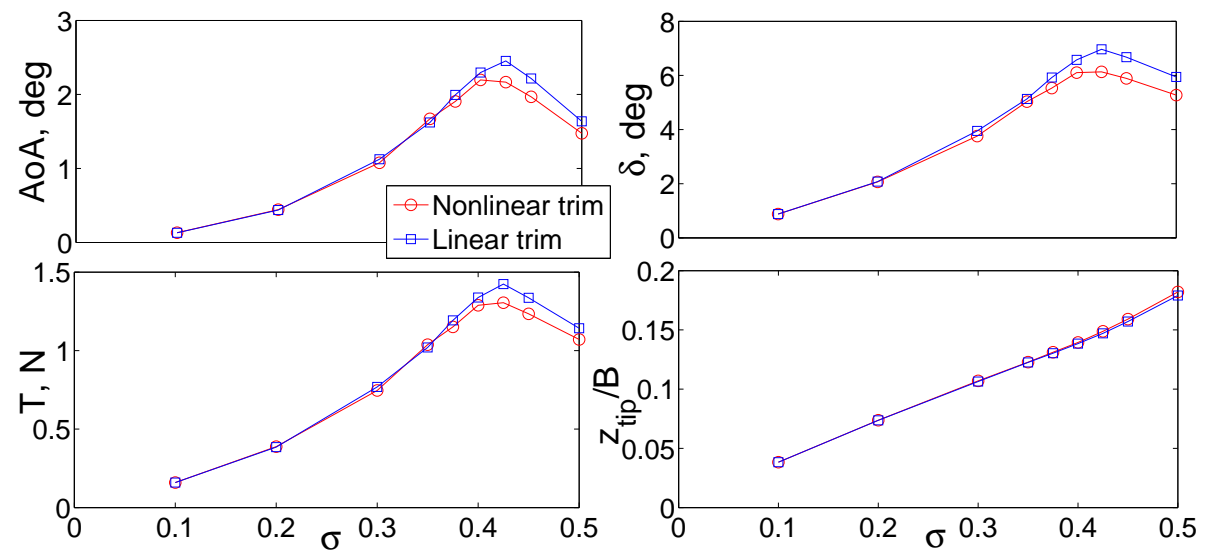

Figure 9. Trim characteristics of the model HALE aircraft as a function of the stiffness of the main wing. Nondimensional tip deflection of the main wing, $z_{t i p} / B$ is presented at trim conditions, together with the corresponding inputs in angle of attack, AoA, elevator deflection, $\delta$, and thrust per propeller, $T$.
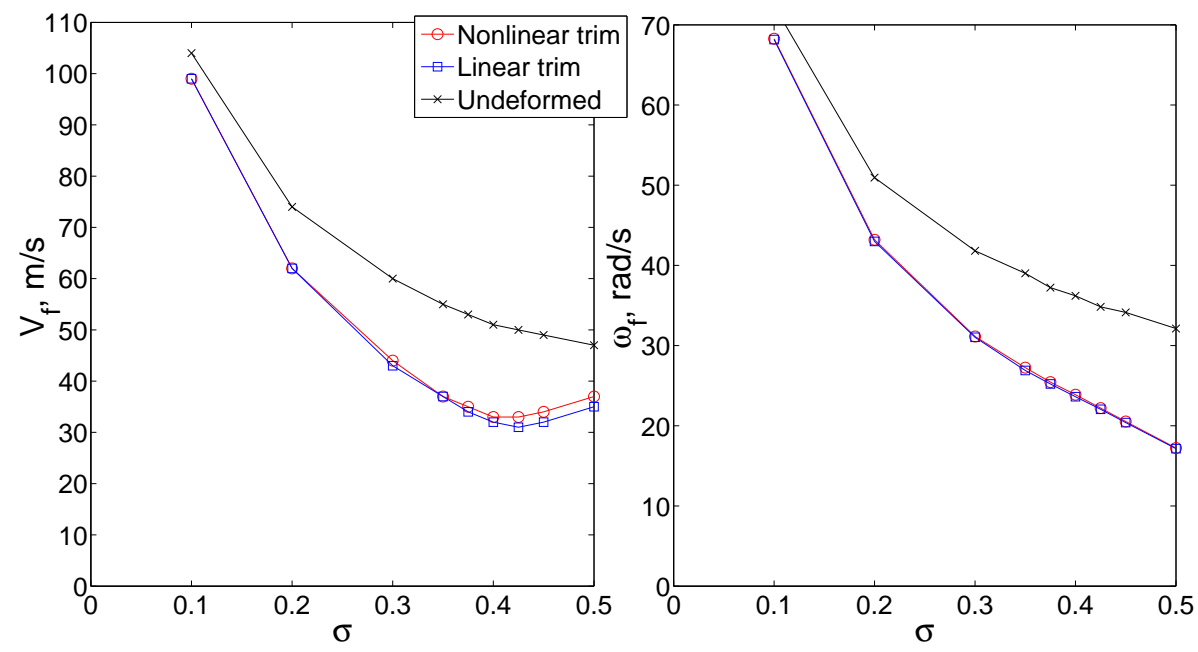

Figure 10. Flutter speed and frequency of the model HALE aircraft as a function of the stiffness of the main wing. Undeformed configuration, linear trim and nonlinear trim.

Flutter speeds and frequencies are shown in Figure 10. As one should expect, linearizing around the undeformed configuration leads to a significant overprediction of the flutter onset on a very flexible aircraft, which may lead to catastrophic consequences. In fact, even for a relatively stiff wing, $\sigma=0.1$, which corresponds to a tip deflection of the order of $4 \%$ of the wing semi-span, the discrepancy is already noticeable. As the stiffness decreases this difference increases dramatically $(\approx 60 \%$ error at $\sigma=0.425)$, until a minimum is found for the flutter curves of the deformed aircraft. As mentioned above, this minimum corresponds to the maximum found in the trim variables. As expected, while the tip deflection remains below $15 \%$ of the wing semi-span, both linear and nonlinear flutter results match. However, as the deformations increase, a small change in the flutter speed becomes apparent, even though the frequency remains nearly the same. Obviously, the departure coincides with the shift in trim conditions. In this case, as opposed to the linearization around the undeformed configuration, the estimate of instability onset is slightly conservative. Finally, note that results beyond these values of $\sigma$ are not presented because trimming the aircraft becomes impossible, and this in fact sets an upper boundary to the achievable flight velocities. 


\section{Open-Loop Response in Time Domain}

\section{Roll response in high-aspect ratio wings}

In this section we examine the roll response of a single high-aspect ratio straight wing using ailerons. This simple problem allows the comparison of the results obtained from SHARP with analytical solutions based on strip theory. The problem is limited to twist flexibility only, assuming very large bending stiffness, and to zero rigid AoA with negligible gravitational effects. The aerodynamic and elastic properties of the wing are presented in Table 5. The problem is solved using the dynamic module in SHARP with unsteady aerodynamics and nonlinear flexible-body dynamics. The ailerons are modeled as full span control surfaces at the trailing edge of each flat wing. In order to understand the effect of flexibility on the response of the wing, results are compared to the rigid-body solution of the problem.

Table 5. Properties of the high-aspect ratio wing for roll response

\begin{tabular}{ll}
\hline \hline Chord, $c$ & $0.2 \mathrm{~m}$ \\
Semi-span, $B$ & $10 \mathrm{~m}$ \\
Aileron chord, $c_{f}$ & $20 \%$ chord \\
Elastic axis (from l.e.) & $50 \%$ chord \\
Center of gravity (from l.e.) & $50 \% \mathrm{chord}$ \\
Body moment of inertia in roll, $I_{\phi}$ & $100 \mathrm{~kg} \cdot \mathrm{m}$ \\
Sectional torsional stiffness, $G J$ & $100 \mathrm{~N} \cdot \mathrm{m}^{2}$ \\
Sectional bending stiffness & $\infty$ \\
\hline \hline
\end{tabular}

An analytical model can be easily obtained for this problem using strip theory without tip corrections. First, the lift forces and pitching moments per unit length created by the aerodynamic loads about the aerodynamic center (on the right wing with positive roll rate) can be approximated, assuming zero rigid AoA, as

$$
\begin{aligned}
\frac{d L}{d y} & =q_{\infty} c\left[c_{l, \alpha}\left(\theta+\frac{y \dot{\phi}}{V_{\infty}}\right)-c_{l, \beta} \beta^{*}\right], \\
\frac{d M_{a c}}{d y} & =-q_{\infty} c^{2} c_{m, \beta} \beta^{*},
\end{aligned}
$$

where $q_{\infty}=\frac{1}{2} \rho_{\infty} V_{\infty}$ is the free-stream dynamic pressure, $\phi$ is the roll angle, and the aileron deflection is $\beta^{*}>0$ for positive roll (right wing moving upwards). Assuming negligible structural dynamic effects in the deformation of the wing under these loads, and very large bending stiffness and negligible gravitational effects, as mentioned above, the twist angle at each point $y$ along the wing can be found as

$$
\theta=(1-\tan \lambda B \sin \lambda y-\cos \lambda y) \nu \beta^{*}-\left(\lambda y-\frac{\sin \lambda y}{\cos \lambda B}\right) \frac{\dot{\phi}}{\lambda V_{\infty}},
$$

with

$$
\lambda^{2}=\frac{q_{\infty} e c c_{l, \alpha}}{G J}, \quad \nu=\frac{c c_{m, \beta}+e c_{l, \beta}}{e c_{l, \alpha}} .
$$

where the distance between elastic axis and aerodynamic center is $e=0.25$. From Newton's law, the angular acceleration of the aircraft is $I_{\phi} \ddot{\phi}=M_{\phi}$, with $I_{\phi}$ being the mass moment of inertia of the wing about the $x$ axis and the roll moment given as

$$
M_{\phi}=-2 \int_{0}^{B} y d L=q_{\infty} c B^{2}\left[C_{\beta} \beta^{*}-C_{\dot{\phi}} \frac{\dot{\phi}}{\lambda V_{\infty}}\right],
$$

The nondimensional constants $C_{\beta}$ and $C_{\dot{\phi}}$ can be obtained through integration of Eq. (42). This results in a second order differential equation of motion for the roll angle $\phi$ that can be easily integrated. Figure 11 shows the roll angle for $V_{\infty}=5 \mathrm{~m} / \mathrm{s}, \rho_{\infty}=1 \mathrm{~kg} / \mathrm{m}^{3}$ and $\beta^{*}=0.1 \mathrm{rad}$. Results compare those from the 
analytical model with the results from SHARP with a constant time step of $d t=0.005 \mathrm{~s}$. The aerodynamic coefficients for the analytical model corresponds to those of a flat plate and are $c_{l, \alpha}=5.8854, c_{l, \beta}=4.1053$ and $c_{m, \beta}=0.64$.

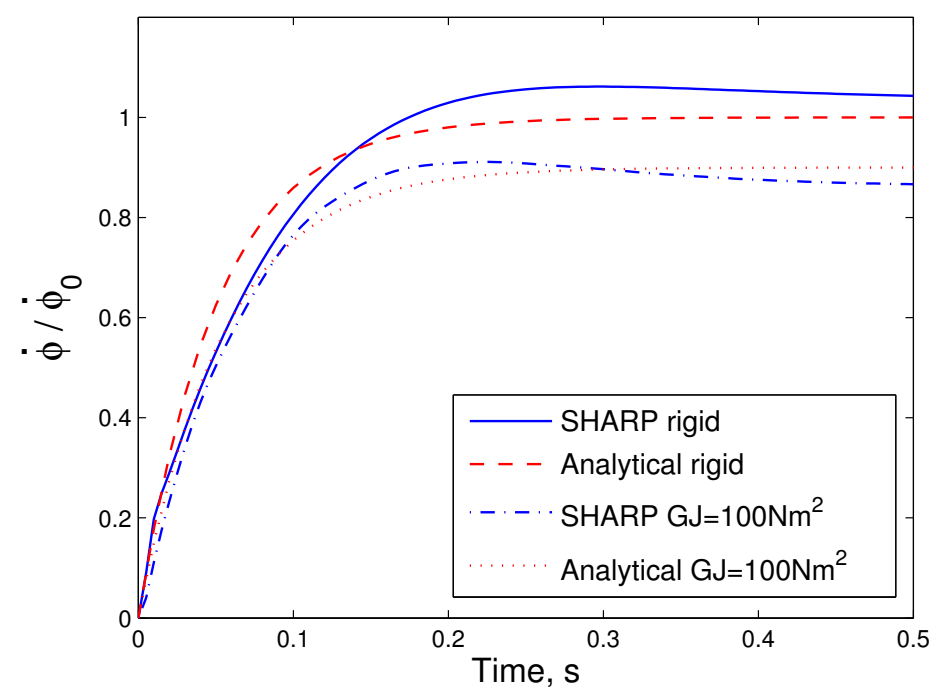

Figure 11. Roll rate in the open-loop response of the wing defined in Table 5 to a constant aileron deflection $\beta^{*}=0.1$ rad. Results are normalised with steady-state roll rate of the analytical solution for a rigid wing, $\dot{\phi}_{0}$.

From the results, it is evident that, after a short transient part, a steady state response is reached and the wing rolls at a constant angular velocity. It also shows a decrease in roll rate due to twisting of the wing, as the aerodynamic forces are obtained for the deformed configuration at each time step resulting in less lift acting perpendicular to the wing. Good comparison is found between the analytical prediction and the numerical results obtained in SHARP for both rigid and flexible wings. The small differences between the analytical solution and SHARP for the steady state response are mainly due to the wing tip effects and induced drag, which are neglected in the analytical solution. Also, the aerodynamic module in SHARP captures unsteady effects, which explains the differences in slope during the transient.

\section{Pitch response of HALE aircraft}

This section presents some exploratory studies of the open-loop time-domain response of the generic HALE vehicle of Figure 8. As in the stability analysis in section V.C, the stiffness property of the main wing is used as a parameter $(\sigma$, in Table 4$)$ to understand the effect of flexibility on the flight dynamic response of the aircraft. The trimmed aircraft is subject to a disturbance in the form of a commanded input on the elevators.

Firstly, the aircraft is trimmed dynamically for a free stream velocity of $25 \mathrm{~m} / \mathrm{s}$ and different values of $\sigma$. The free stream velocity was chosen such that the aircraft is deforming moderately (within the linear elastic regime) with the upper boundary being the flutter speed for the range of analyzed values of $\sigma$ shown in Figure 10. Three studies have been conducted to explore the capabilities of the linear dynamic solution in SHARP: flexible model with (i) $\sigma=10^{-5}$, (ii) $\sigma=0.2$ and (iii) $\sigma=0.5$. In each case a static analysis is carried out first to obtain the deformed shape of the aircraft under trimmed, steady flight conditions. The aircraft is subsequently disturbed around dynamic equilibrium through a (1-cos) doublet elevator input defined in Figure 12 (a) with the excitation amplitude of $\Delta \delta_{\max }=5 \mathrm{deg}$ (equal elevator deflection on right and left tail) and $T=1 \mathrm{~s}$. For all cases the dynamic response is computed using the linearized form of the coupled set of dynamic equations with a time step size of $d t=0.02 \mathrm{~s}$.

The response of the aircraft is shown in Figures 12 (b-d) in terms of the translational and rotational velocity components of the origin of the body-fixed reference system $a$ expressed in the inertial frame $G$.

Comparing the solution of the linearized models for different values of $\sigma$, it can be seen that flexibility has an effect on the flight dynamics of the vehicle resulting in an increased amplitude of oscillation for larger values of $\sigma$ during the doublet maneuver. The flexibility effect will have to be investigated further 

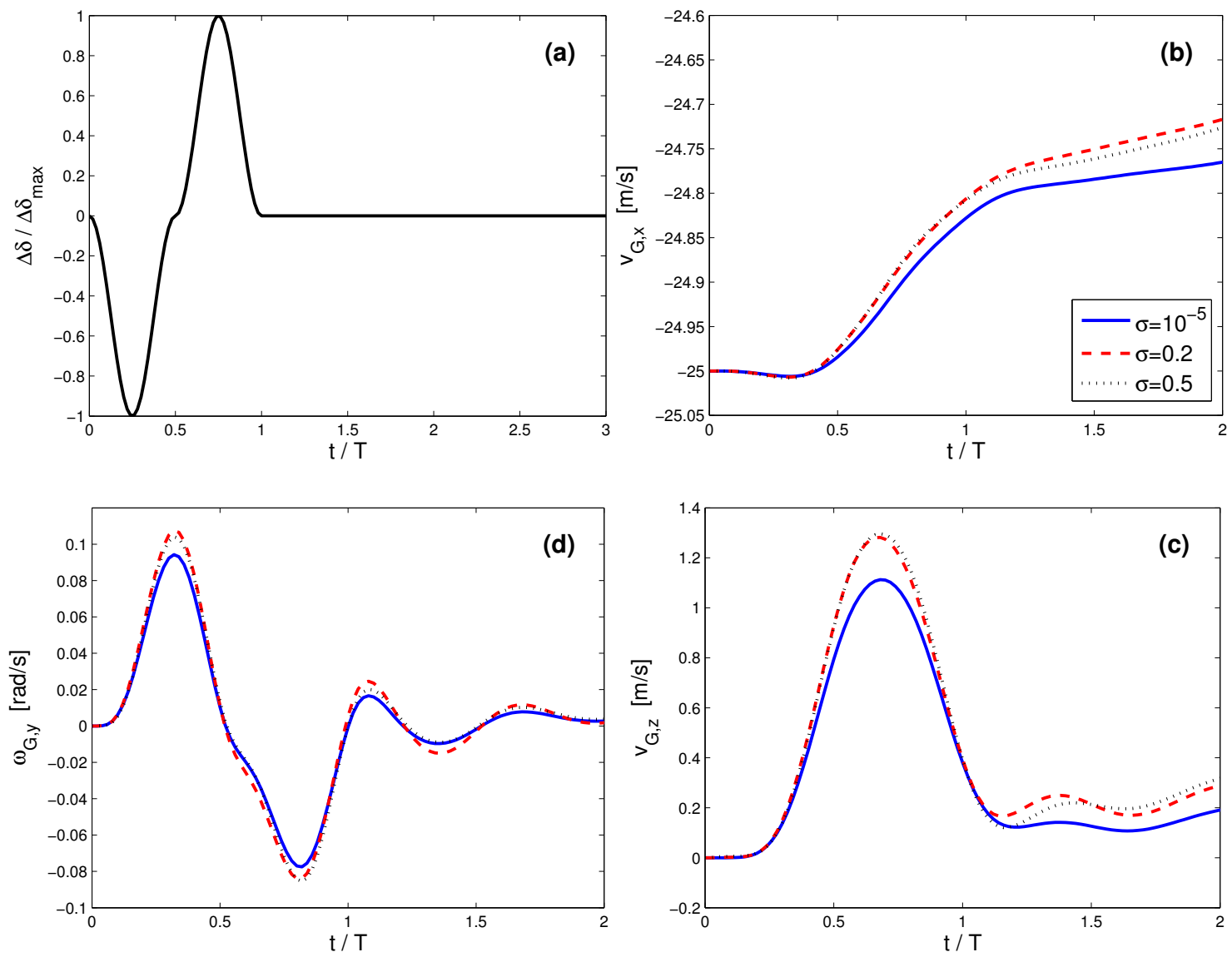

Figure 12. Linear open-loop transient response of the HALE vehicle of Figure 8 subject to elevator doublet input (a). Time history of (b) longitudinal linear velocity $v_{G, x}$, (c) vertical linear velocity $v_{G, z}$ and (d) angular velocity $\omega_{G, y}$ of the body-fixed reference frame $a$ expressed in the inertial frame $G$.

for larger structural deformations using the fully-coupled nonlinear flexible model. Future studies will focus on the contribution of geometrically-nonlinear deformations on the flight dynamics of very flexible aircraft undergoing large rigid-body motion comparing a broader range of excitation amplitudes $\Delta \delta_{\max }$.

\section{Wake interference effects}

Finally, the impact of the interference effects will be assessed. The model HALE aircraft described in Table 4 and Figure 8 has been considered again, with $\sigma=0.2$. In order to study the influence between the wake shed by the main wing and the tail, a sinusoidal elevator deflection is commanded around the trim configuration, and the time-domain response of the aircraft is monitored. This analysis is an open-loop version of that performed in our previous work: ${ }^{9}$ instead of prescribing the motions of the aircraft, a maneuver is commanded and the vehicle is free to follow the trajectory that will result from this input - note that no particular path has been sought for, but this represents a simple example to analyze the interference between the wing wake and the tail.

The elevator perturbation will be given by

$$
\delta=\delta_{0}+\delta^{*} \sin (\omega t)
$$

where $\delta_{0}=9.85 \mathrm{deg}$ is the elevator deflection for the trimmed aircraft, and the oscillation frequency is $\omega=5$ $\mathrm{rad} / \mathrm{s}$, close to the first bending mode of the main wing, $\omega_{b 1}=5.09 \mathrm{rad} / \mathrm{s}$. $\delta^{*}$ can take both positive and negative values, and these have been chosen so that interference effects become visible, i.e., by making the wake pass close to the tail, but avoiding direct collisions. 


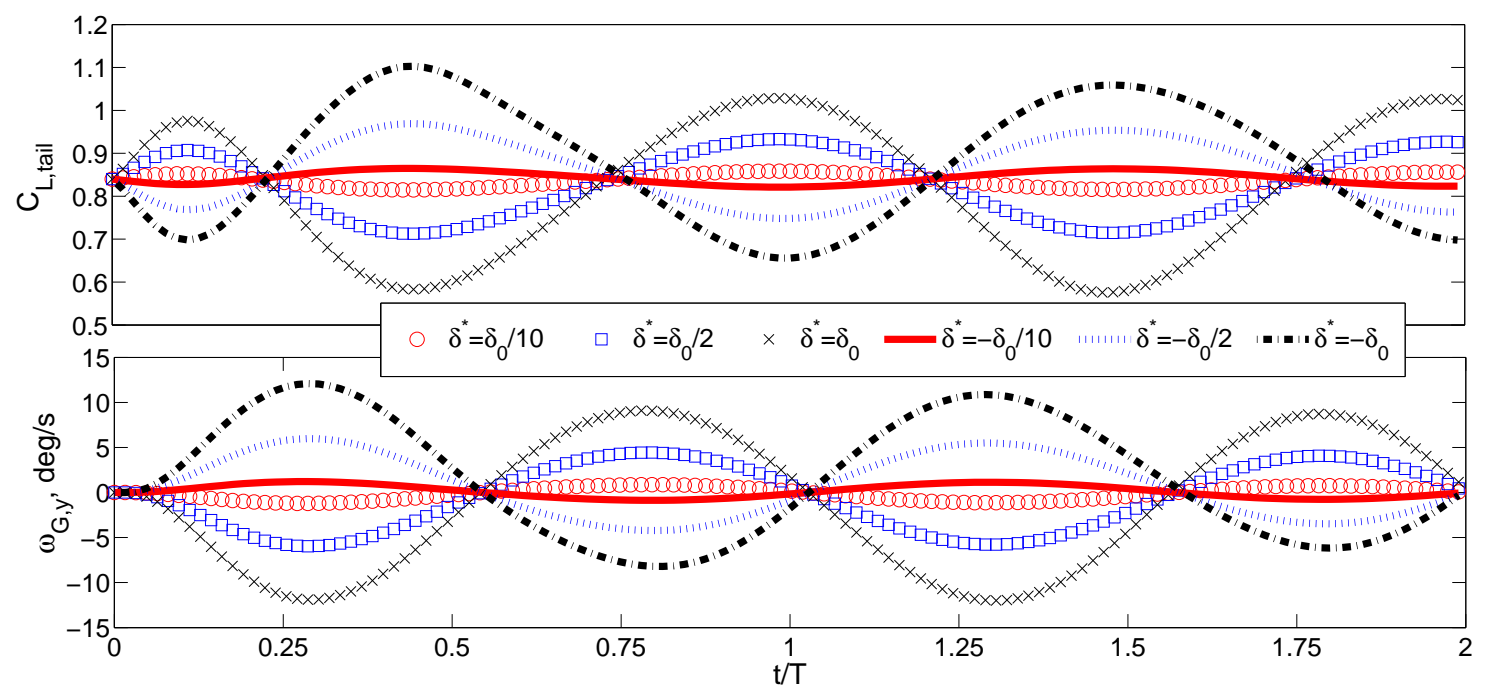

Figure 13. Tail lift coefficient and aircraft pitch rate for sinusoidal elevator input of different amplitudes. Model HALE aircraft described in Table 4 and Figure 8 with $\sigma=0.2$.

Figure 13 presents the lift coefficient of the T-tail of the aircraft and the pitch rate for different values of $\delta^{*}$. The first two cycles of the commanded sinusoidal elevator deflection are depicted. For positive values of $\delta^{*}$, the lift at the tail starts increasing at the beginning of the perturbation, and hence the aircraft starts pitching down. The opposite happens for $\delta^{*}<0$. For small amplitudes around the trim value, the lift coefficient of the tail follows a sinusoidal evolution, consistent with the pitching motion of the aircraft. As $\delta^{*}$ increases, however, the effect of the wake interference becomes apparent, as the lift curve is not a perfect sinusoid anymore. This is especially obvious with $\delta^{*}= \pm \delta_{0}$, for $\frac{T}{2}<t<\frac{3}{4} T$. This behavior corresponds to a situation in which the wake gets close enough to the tail, when the distance is smaller than a chord length. ${ }^{9}$

Note that after these two oscillatory cycles a residual pitch rate persists (barely visible in Figure 13), and hence, the perturbation on the elevator yields a resultant motion that departs from the trim state. Note also that for the maximum values of $\delta^{*}$, the elevator deflection exceeds $19 \mathrm{deg}$, which might lead to flow separation.

Figure 14 presents snapshots of the main wing, T-tail and corresponding wakes of the HALE model aircraft during the first two cycles of an elevator input with $\delta^{*}=-\delta_{0}$. As can be inferred, the wake has not been rolled up in this study. This is motivated by the results obtained in previous exercises with prescribed motions, ${ }^{9}$ where it was found that free wake effects had a negligible impact for this particular geometry and conditions, unless direct wake-tail collisions occur. As this is not the subject of study of this paper, a prescribed wake has been assumed around the trimmed aircraft configuration in order to reduce the computational burden.

\section{Conclusions}

This paper has presented a computational framework for the medium-fidelity modeling and simulation of low-speed flexible aircraft dynamics. The main novelty is in the use of a unsteady vortex lattice aerodynamic model with a free wake and in the use of the rotation vector as independent degree of freedom to describe large structural rotations. This yields a representation which captures the effect of large geometry changes, both in the structural and in the 3-D aerodynamics, but that also coincides with the conventional linear models for small amplitude dynamics. A loosely-coupled time-marching integration scheme has been implemented and exercised on some typical cases, as well as a monolithic state-space representation of the full-system linearized dynamics. This state-space model gives a procedure to obtain the stability characteristics on the original systems states without any need of projecting the structural dynamics on a modal space or pre-computing aerodynamic forces at different frequencies. Note that, for linear problems, the fidelity of the unsteady vortex-lattice is comparable to that of the doublet lattice method. For geometrically-nonlinear deformations, however, the UVLM provides a more faithful representation: 1) the wake shed is non-planar 


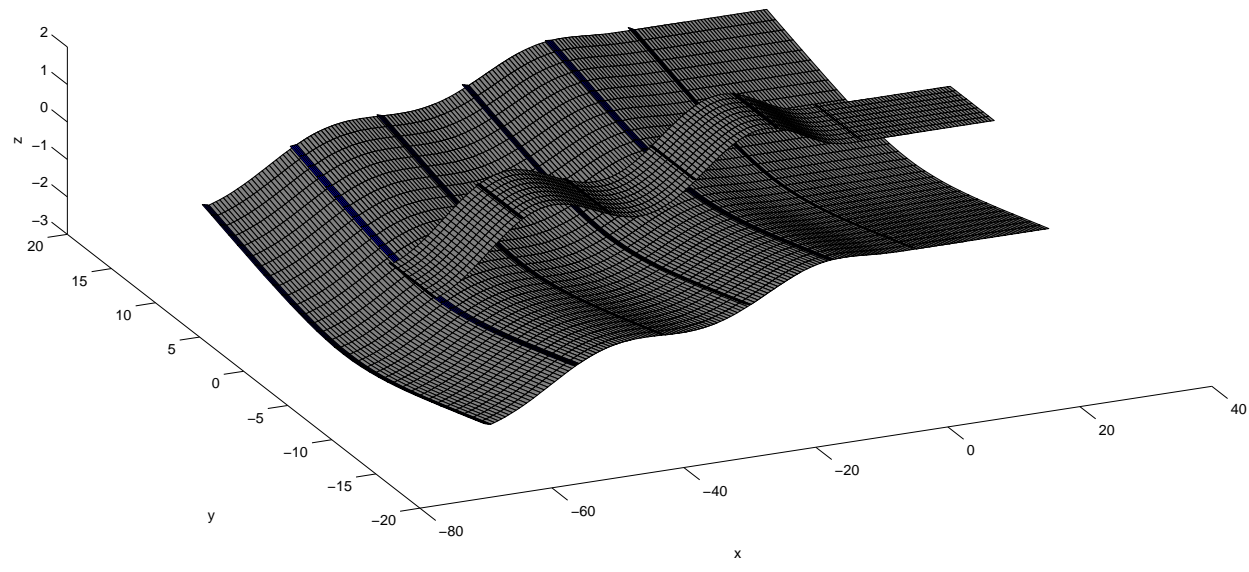

Figure 14. Snapshots of the flight trajectory and shed wake of the model HALE aircraft. $0 \leq t \leq 2 T$ and $\delta^{*}=+\delta_{0}$. Axes have different scale in order to illustrate the oscillations of the vehicle.

and it can be allowed to roll up; 2) the non-penetration boundary condition is enforced on the deformed geometry; and 3) in-plane deformations are captured.

The different solution approaches within this simulation tool have been verified against other methods. In particular, the coupled flexible-body dynamics solver, the static aeroelastic solver, the trim module and the flexible aircraft dynamics solver have been exercised. Results have shown the flexibility of the methodology to analyze quite generic configurations with a minimal modeling effort and modest computational costs. Although the procedure has been described in the paper, some effort is still required for the solution of the nonlinear dynamic response to gusts and atmospheric turbulence. Those have been shown to be major drivers in the design of very large lightweight vehicles and require further attention, in particular with respect to the impact in wing-tail interference. The current model is expected to provide an appropriate framework for the analysis of those situations.

\section{Appendix}

This section shows detailed expressions for the evaluation of the different terms of the flexible body dynamics equations of motion presented in Eq. (31). We first define the following variables to simplify the notation,

$$
\hat{f}^{S}=\left\{\begin{array}{c}
f_{B} \\
m_{B}
\end{array}\right\}, \hat{V}=\left\{\begin{array}{c}
V_{B} \\
\Omega_{B}
\end{array}\right\}, \hat{F}=\left\{\begin{array}{c}
F_{B} \\
M_{B}
\end{array}\right\}, \quad \hat{f} R=\left\{\begin{array}{c}
\mathbb{F}_{a} \\
\mathbb{M}_{a}
\end{array}\right\}, \hat{v}=\left\{\begin{array}{c}
v_{a} \\
\omega_{a}
\end{array}\right\} .
$$

The structural and rigid-body components to the gyroscopic, elastic and external forces in Eq. (31) can be identified as

$$
Q_{g y r}=\left\{\begin{array}{c}
Q_{g y r}^{S} \\
Q_{g y r}^{R}
\end{array}\right\}, \quad Q_{s t i f}=\left\{\begin{array}{c}
Q_{s t i f}^{S} \\
0
\end{array}\right\}, \quad Q_{e x t}=\left\{\begin{array}{c}
Q_{e x t}^{S} \\
Q_{e x t}^{R}
\end{array}\right\} .
$$

The discretized generalized forces for the elastic displacements are given by

$$
\begin{aligned}
Q_{\text {stif }}^{S} & =\int_{0}^{l}\left(N^{T} \Upsilon^{T} A_{K}+N^{T} \Upsilon^{\prime T}+N^{\prime T} \Upsilon^{T} D_{S}^{T}\right) \hat{F} d s \\
Q_{e x t}^{S} & =\int_{0}^{l} N^{T} \Upsilon^{T} D_{S}^{T} \hat{f}^{S} d s
\end{aligned}
$$


The gyroscopic forces can be written as

$$
\begin{aligned}
& Q_{g y r}^{S}=\int_{0}^{l} N^{T} \Upsilon^{T} D^{T}\left(M \hat{V}_{g y r}+A_{\Omega \Omega} M \hat{V}\right) d s, \\
& Q_{g y r}^{R}=\int_{0}^{l} A_{R C} M \hat{V}_{g y r}+A_{\omega R C} M \hat{V} d s .
\end{aligned}
$$

with

$$
\begin{aligned}
A_{\omega R C} & =\left[\begin{array}{cc}
C^{a B} \tilde{\Omega}_{B} & 0 \\
\tilde{R}_{a} C^{a B} \tilde{\Omega}_{B}+\left(\dot{\tilde{R}}_{a}+\tilde{\omega}_{a} \tilde{R}_{a}-\tilde{R}_{a} \tilde{\omega}_{a}\right) C^{a B} & C^{a B} \tilde{\Omega}_{B}
\end{array}\right], \\
\hat{V}_{g y r} & =\left\{\begin{array}{c}
C^{B a} \tilde{\omega}_{a} \dot{R}_{a}+\tilde{V}_{B} T \dot{\Psi} \\
\left(\dot{T}+\tilde{\Omega}_{B} T\right) \dot{\Psi}
\end{array}\right\} .
\end{aligned}
$$

The tangent mass matrices have been defined as a function of the deformed state, $q$, as

$$
M=\left[\begin{array}{ll}
M^{S S} & M^{S R} \\
M^{R S} & M^{R R}
\end{array}\right],
$$

with

$$
\begin{aligned}
M^{S S} & =\int_{0}^{l} N^{T} \Upsilon^{T} D^{T} M D \Upsilon N d s, \\
M^{S R} & =\int_{0}^{l} N^{T} \Upsilon^{T} D^{T} M\left(A_{R C}\right)^{T} d s, \\
M^{R R} & =\int_{0}^{l} A_{R C} M\left(A_{R C}\right)^{T} d s,
\end{aligned}
$$

with

$$
A_{R C}=\left[\begin{array}{cc}
C^{a B} & 0 \\
\tilde{R}_{a} C^{a B} & C^{a B}
\end{array}\right] .
$$

Finally, the external generalized forces on the rigid-body equation are

$$
Q_{e x t}^{R}=D_{R}^{T} \hat{f}^{R} .
$$

In the previous expressions, the following matrices were used

$$
\begin{aligned}
& D_{S}=\left[\begin{array}{cc}
C^{B a} & 0 \\
0 & I
\end{array}\right], D_{R}=\left[\begin{array}{cc}
C^{a G} & 0 \\
0 & I
\end{array}\right], A_{\Omega \Omega}=\left[\begin{array}{cc}
\tilde{\Omega}_{B} & 0 \\
\tilde{V}_{B} & \tilde{\Omega}_{B}
\end{array}\right], \\
& A_{K}=\left[\begin{array}{cc}
0 & 0 \\
-\left(\tilde{e}_{1}+\tilde{\gamma}\right) & -\tilde{K}_{B}
\end{array}\right], A_{\omega \omega}=\left[\begin{array}{cc}
\tilde{\omega}_{a} & 0 \\
0 & \tilde{\omega}_{a}
\end{array}\right],
\end{aligned}
$$

and

$$
\Upsilon=\left[\begin{array}{cc}
I & 0 \\
0 & T(\Psi)
\end{array}\right], \quad \Upsilon^{\prime}=\left[\begin{array}{cc}
0 & 0 \\
0 & T^{\prime}(\Psi)
\end{array}\right]
$$

\section{Acknowledgments}

Joseba Murua gratefully acknowledges the financial support provided by the Department of Education, Universities and Research of the Basque Government. The work of Henrik Hesse is sponsored by the UK Engineering and Physical Sciences Research Council. 


\section{References}

${ }^{1}$ Drela, M., "Integrated simulation model for preliminary aerodynamic, structural, and control-law design of aircraft," 40th AIAA/ASME/ASCE/AHS/ASC Structures, Structural Dynamics and Materials Conference, AIAA Paper 1999-1394, St. Louis, Missouri, USA, April 1999.

${ }^{2}$ Patil, M. J., Hodges, D., and Cesnik, C., "Nonlinear Aeroelastic Analysis of Complete Aircraft in Subsonic Flow," Journal of Aircraft, Vol. 37, No. 5, 2000, pp. 753-760.

${ }^{3}$ Patil, M. J., Hodges, D. H., and Cesnik, C. E. S., "Nonlinear Aeroelasticity and Flight Dynamics of High-Altitude Long-Endurance Aircraft," Journal of Aircraft, Vol. 38, No. 1, 2001, pp. 88-94.

${ }^{4}$ Wang, Z., Chen, P. C., Liu, D. D., Mook, D. T., and Patil, M. J., "Time Domain Nonlinear Aeroelastic Analysis for HALE Wings," 47th AIAA/ASME/ASCE/AHS/ASC Structures, Structural Dynamics, and Materials Conference, AIAA Paper 2006-1640, Newport, RI, USA, May 2006.

${ }^{5}$ Shearer, C. and Cesnik, C., "Nonlinear Flight Dynamics of Very Flexible Aircraft," Journal of Aircraft, Vol. 44, No. 5, 2007, pp. 1528-1545.

${ }^{6} \mathrm{Su}, \mathrm{W}$. and Cesnik, C., "Nonlinear Aeroelasticity of a Very Flexible Blended-Wing-Body Aircraft," Journal of Aircraft, Vol. 47, No. 5, 2010, pp. 1539-1553.

${ }^{7}$ Palacios, R., Murua, J., and Cook, R., "Structural and Aerodynamic Models in the Nonlinear Flight Dynamics of Very Flexible Aircraft," AIAA Journal, Vol. 48, No. 11, November 2010, pp. 2648-2559.

${ }^{8}$ Elzebda, J. M., Mook, D. T., and Nayfeh, A. H., "Numerical Simulations of Steady and Unsteady Vorticity-Dominated Aerodynamic Interference," Journal of Aircraft, Vol. 31, No. 5, 1994, pp. 1031-1036.

${ }^{9}$ Murua, J., Palacios, R., and Graham, J., "Modeling of Nonlinear Flexible Aircraft Dynamics Including Free-Wake Effects," AIAA Guidance, Navigation, and Control Conference, AIAA Paper 2010-8226, Toronto, Ontario, Canada, August 2010.

${ }^{10} \mathrm{Katz}$, J. and Plotkin, A., Low-Speed Aerodynamics, Cambridge Aerospace Series, Cambridge University Press, New York, NY, USA, 2nd ed., 2001.

${ }^{11}$ Albano, E. and Rodden, W. P., "A Doublet-Lattice Method for Calculating Lift Distributions on Oscillating Surfaces in Subsonic Flow," AIAA Journal, Vol. 7, No. 2, 1969, pp. 279-285.

${ }^{12}$ Rodden, W. P., "The Development of the Doublet-Lattice Method," International Forum on Aeroelasticity and Structural Dynamics, Rome, Italy, June 1997.

${ }^{13}$ Fritz, T. E. and Long, L. N., "Object-oriented unsteady vortex lattice method for flapping flight," Journal of Aircraft, Vol. 41, No. 6, November-December 2004, pp. 1275-1290.

${ }^{14}$ Leishman, J. G., Principles of Helicopter Aerodynamics, Cambridge Aerospace Series, Cambridge University Press, 2nd ed., 2006.

${ }^{15}$ Dovgiǐ, S. and Shekhovtsov, A., "An Improved Vortex Lattice Method for Nonstationary Problems," Journal of Mathematical Sciences, Vol. 104, No. 6, May 2001, pp. 1615-1627.

${ }^{16}$ Wie, S. Y., Lee, S., and Lee, D. J., "Potential Panel and Time-Marching Free-Wake Coupling Analysis for Helicopter Rotor," Journal of Aircraft, Vol. 46, No. 3, May-June 2009, pp. 1030-1041.

${ }^{17}$ Hodges, D., "A mixed variational formulation based on exact intrinsic equations for dynamics of moving beams," International Journal of Solids and Structures, Vol. 26, No. 11, 1990, pp. 1253-1273.

${ }^{18}$ Géradin, M. and Cardona, A., Flexible Multibody Dynamics: A Finite Element Approach, John Wiley \& Sons, Inc., 2001.

${ }^{19}$ Palacios, R. and Cesnik, C., "Cross-Sectional Analysis of Non-Homogeneous Anisotropic Active Slender Structures," AIAA Journal, Vol. 43, No. 12, 2005, pp. 2624-2638.

${ }^{20}$ Stevens, B. L. and Lewis, F. L., Aircraft Control and Simulation, John Wiley \& Sons, Inc., New York, NY, USA, 1992.

${ }^{21}$ Crisfield, M. A. and Jelenić;, G., "Objectivity of strain measures in the geometrically exact three-dimensional beam theory and its finite-element implementation," Proceedings of the Royal Society of London. Series A: Mathematical, Physical and Engineering Sciences, Vol. 455, No. 1983, 1999, pp. 1125-1147.

${ }^{22}$ S. Thepvongs, S., Cesnik, C., Palacios, R., and Peters, D., "Finite-State Aeroelastic Modeling of Rotating Wings with Deformable Airfoils," American Helicopter Society 64th Annual Forum and Technology Display, Montreal, Canada, May 2008.

${ }^{23}$ Hall, K., "Eigenanalysis of Unsteady Flows about Airfoils, Cascades and Wings," AIAA Journal, Vol. 32, No. 12, 1994, pp. 2426-2432.

${ }^{24}$ Goland, M., "The flutter of a uniform cantilevered wing," Journal of Applied Mechanics, Vol. 12, No. 4, 1945, pp. A197A198.

${ }^{25}$ ZONA Technology, Inc., Scottsdale, AZ, USA, ZAERO User's Manual. 